\title{
ÉTUDE DU RÉGIME TRANSITOIRE \\ DE L'ÉCOULEMENT DE L'EAU \\ PENDANT LE DRAINAGE DES NAPPES \\ A SURFACE LIBRE
}

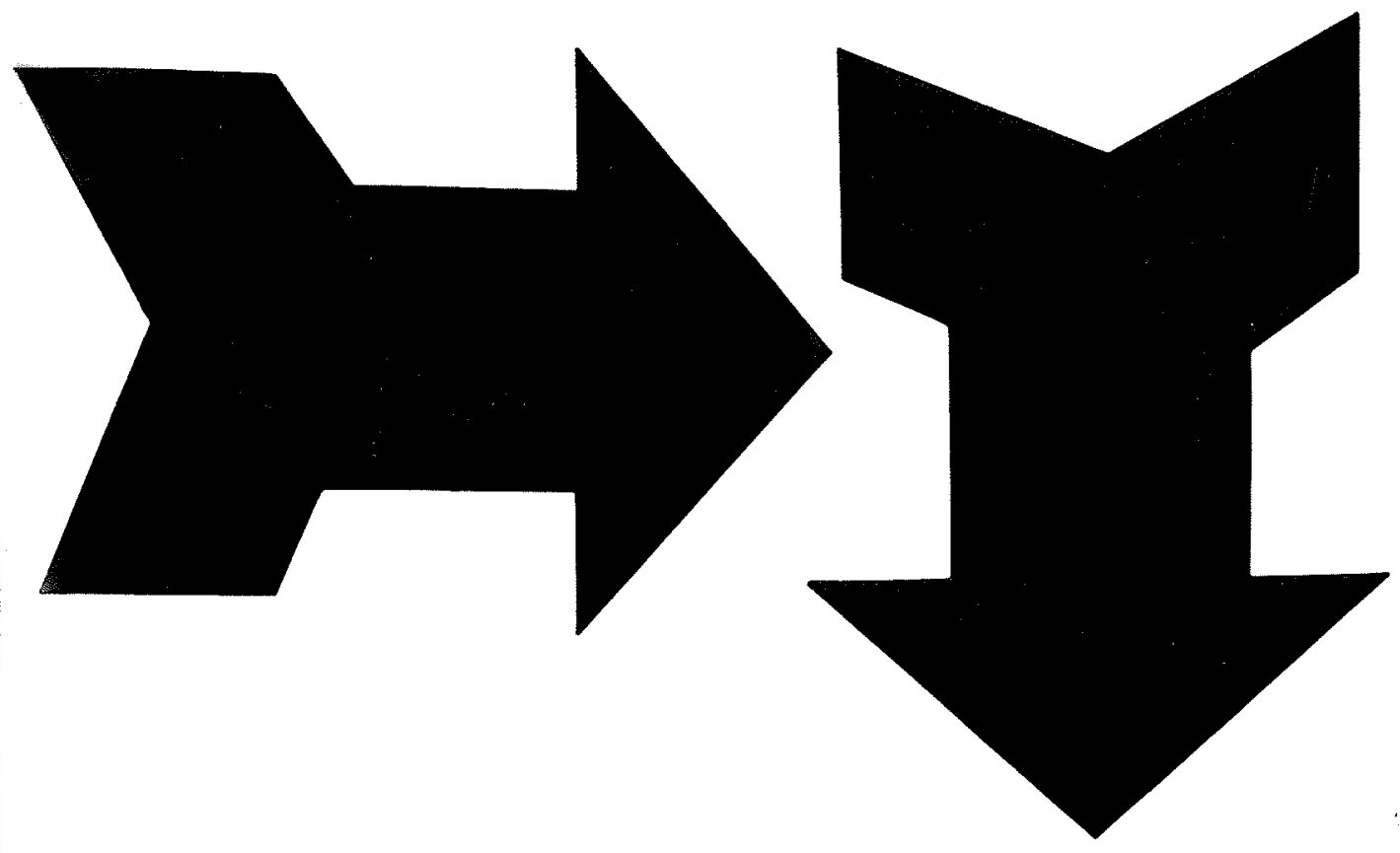

PAR G. VAChAUD *

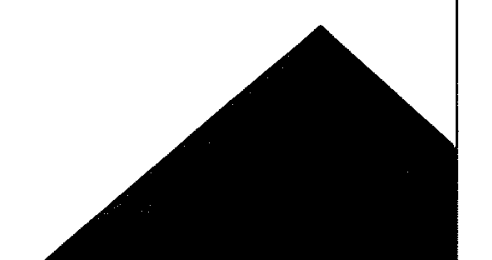

\section{I. - Introduction}

Le drainage des nappes à surface libre a longlemps été considéré comme un problème d'écoulement simple, répondant à l'hypothèse suivante: lors du rabattement d'une nappe, il y a drainage instantané de la partie de sol située au-dessus du niveau de la nappe (Ferris, 1962; Hantush, 1966). Cette hypothèse conduit à la détermination de la porosité effective, ou porosité de drainage (specific yield) définie par :

$$
\varepsilon=\theta_{0}-\theta_{r}
$$

où $\theta_{0}$ est la teneur en eau du sol sous le niveau de la nappe, $\theta_{r}$ est la teneur en eau résiduelle représenlant la quantité d'eau restant fixée aux pores par des forces d'absorption.

Lors d'un rabattement $\Delta Z$ d'une nappe, le volume d'eau drainé est alors directement obtenu par :

$$
\Delta V_{w}=\varepsilon \cdot A \cdot \Delta Z
$$

où A est la surface de la nappe. Ia position du niveau de la nappe est calculée à partir des écuations de l'hydrodynamique des milieux poreux saturés. Pour un écoulement à deux dimensions, entre deux drains parallèles, on a ainsi :

$$
\mathbf{I}_{n}\left[\frac{\partial}{\partial x}\left(h \frac{\partial h}{\partial x}\right)+\frac{\partial}{\partial y}\left(h \frac{\partial h}{\partial y}\right)\right]=\varepsilon \frac{\partial h}{\partial t}
$$

\footnotetext{
* Attaché de Recherches au C.N.R.S., Isaboratoire de Mécanique des Fluides, Grenoble.
}

où : $\quad h$ est la cote de la surface libre;

$\mathbf{K}_{0} \quad$ la perméabilité du sol saturé.

Après linéarisation, cetle équation se ramène (Van Schilfgaarde, 1957) à :

$$
\frac{\partial^{2} h}{\partial x^{2}}+\frac{\partial^{2} h}{\partial y^{2}}=\frac{\varepsilon}{\mathrm{K}_{0} m} \frac{\partial h}{\partial t}
$$

où $m$ est la distance entre les drains et le fond de la nappe, reposant sur une couche imperméable.

Bien qu'il n'existe pas d'essais normalisés pour déterminer $\theta_{r}$, on emploie ainsi couramment $\varepsilon$ comme une constante caractéristique des écoulements à surface libre dépendant uniquement de la nature du sol (Todd, 1957; De Wiest, 1965; Bouwer, 1963). Une des méthodes classiques de détermination de $\varepsilon$ revient à prendre un échantillon de sol saturé, à teneur en eau initiale $\theta_{0}$, à le laisser se drainer par gravité, et à mesurer sa teneur en eau $\theta_{r}$ Iorsque le drainage est «terminé » (Meinzer, 1932), sans que ne soient cependant spécifiées ni la hauteur de l'échantillon, ni la durée du drainage, ni limportance du rabattement.

Si l'ón considère (fig. 1 a), le drainage d'un échanlillon de sol de hauteur $\mathbf{L}$, initialement saturé sur toute sa hauteur el si l'on note:

$Z$ le rabattement imposé à la nappe au temps $t=0$ ( $Z$ sera pris comme une grandeur arithmétique);

$z$ la cote d'une section de la colonne, l'origine des cotes étant située à la base de la colonne, avec l'axe $\mathrm{O} z$ orienté vers le haut, 

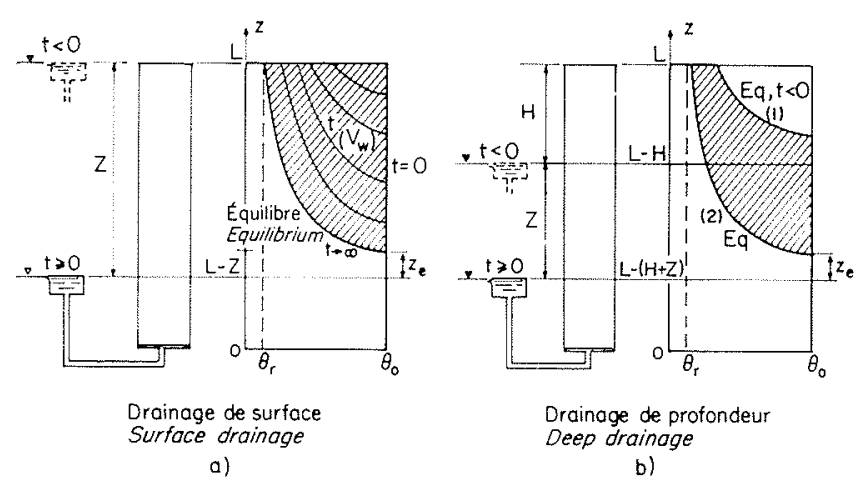

a)

Drainage de profondeur Deep drainage

b)

$1 /$ Schéma des essais de drainage.

Schematic diagram of drainage tests.

$\theta(z, t)$ la valeur de la teneur en eau à la cote $z$ au temps $t$, avec $\theta(z, 0)=\theta_{0}$;

les teneurs en eau dans la colonne vont, au cours du drainage, évoluer entre la répartition initiale $\theta=\theta_{0}$ et la répartition finale donnée par la courbe d'équilibre en drainage $\mathrm{A}$ un instant $t$, le profil hydrique $\theta(z, t)$ permet de calculer le volume d'eau écoulé par la base de la colonne par :

$$
\mathrm{V}_{w}(t)=\mathrm{A} \int_{0}^{\mathrm{L}}\left(\theta_{0}-\theta\right) d z
$$

Le volume drainable maximal, pour un rabattement $Z$ donné, sera directement obtenu par l'aire du triangle curviligne délimité par la courbe de succion en drainage $\psi(\theta)$, la surface du sol et la valeur de $\theta_{0}$. Soit $V_{\infty}$ ce volume limite, obtenu au bout d'un temps très grand (fig. 5). Si l'on définit le coefficient d'emmagasinement « storage coefficient $\gg S$ par :

$$
\mathrm{S}=\frac{V_{w}}{V_{s}}
$$

où $V_{s}$ est le volume de sol drainé, on voit immédiatement que $\mathrm{S}$ est essentiellement une fonction de $\mathrm{Z}$ et $t$.

Si l'on considère enfin le cas général du drainage à partir d'une nappe initialement à la distance $\mathbf{H}$ de la surface du sol (dans le cas donné ci-dessus (fig. $1 a$ ) on a $\mathrm{H}=0$ ), on aboutit à une relation du type :

$$
\mathrm{S}=-f(\mathrm{H}, \mathrm{Z}, t)
$$

avec, dans le cas où $\mathrm{H} \geqslant \mathrm{H}_{\mathrm{C}}$ :

$$
\lim _{t \rightarrow \infty}=\varepsilon
$$

La courbe $\psi(\theta)$ tend vers une assymptote verticale, dans le domaine des faibles teneurs en eau, qui correspond à $\theta_{r} . \mathrm{H}_{c}$ est une profondeur critique de la nappe égale à la succion à partir de laquelle on a pratiquement $\theta=\theta$. (Vachaud, $1967 a$ ).

Le problème du drainage d'une nappe à surface libre doit donc être considéré comme un problème d'écoulement en milieu poreux non saturé, répondant à une équation du mouvement adapté à ce type de problèmes.

Si l'on définit :

$q$ le débit unitaire correspondant à une teneur en eau $\theta$; k la conductivité hydraulique, fonction de la teneur en eau $\theta$;

$\Phi$ le potentiel total formé de deux composantes :

- le potentiel capillaire $\psi$ correspondant à une teneur en eau $\theta$ et donné par la courbe de succion du matériau (c'est une grandeur négative);

- le potentiel gravitaire $z$, correspondant à la cote d'une section de la colonne :

$$
\Phi=\psi+z
$$

(à l'équilibre, $\psi=-z$ ),

l'équation de l'écoulement sera obtenue en considérant :

a) l'équation de continuité :

$$
\operatorname{div} q=-\frac{\partial \theta}{\partial t}
$$

b) la loi de Darcy généralisée :

$$
q=-k \operatorname{grad} \Phi
$$

Pour un écoulement unidimensionnel orienté dans la direction des $z$ décroissants, on obtient, en combinant $(6 a)$ et $(6 b)$, l'équation générale de l'écoulement :

$$
\frac{\partial \theta}{\partial t}=\frac{\partial}{\partial z}\left(k \frac{\partial \psi}{\partial z}\right)+\frac{\partial k}{\partial z}
$$

permettant d'évaluer $\theta(z, t)$ à partir de $k(\theta)$ et $\psi(\theta)$ si ces fonctions sont connues.

Les conditions initiales et aux limites permettant de résoudre cette équation seront différentes pour deux classes de problèmes :

a) drainage d'une nappe initialement de surface $(\mathrm{H}=\mathbf{0}$ ) (fig. 1 $a$ ): la nappe passe de la cote $z=\mathrm{L}, t<0$ à la cote $z=\mathrm{L}-\mathrm{Z}, t \geqslant 0$;

b) drainage d'une nappe profonde $(H \neq 0)$ (fig. 1 b) : la nappe passe de la cote $z=\mathrm{L}-\mathrm{H}, t<0$ à la cote $z=\mathrm{L}-(\mathrm{H}+\mathrm{Z}), t \geqslant 0$. Pour $t<0$ il existe au-dessus de la nappe une zone de teneur en eau variable. Dans le cas illustré figure $1 b$ nous avons supposé que le profil hydrique 1 dans cette zone correspond à un profil d'équilibre. Les profils hydriques évolueront donc dans ce cas pendant le drainage entre les courbes 1 et 2 déduites par translation $Z$.

Précisons enfin que le « niveau» de la nappe est défini par la cote du plan d'eau obtenu dans un récipient de $25 \mathrm{~cm}$ de diamètre relié à la base de la colonne. Cette cote ne correspond donc pas à la limite de la zone de teneur en eau $\theta_{0}$ dans la colonne. En effet, en dessous du niveau de la nappe, le sol est à une teneur en eau uniforme $\theta_{0}$ et l'eau du sol est soumise à une pression supérieure à la pression atmosphérique; au-dessus de la nappe, l'eau du sol est en dépression mais, à l'équilibre, on rencontre d'abord une zone d'épaisseur $z_{e}$ où la teneur en eau vaut $\theta_{0}$ (frange capillaire), puis une zone où la teneur en eau varie progressivement de $\theta_{0} \grave{a} \theta_{r}$. 
On peut apprécier la complexité du problème en examinant le cas simplifié où l'on néglige l'existence de la zone non saturée $\left(\theta<\theta_{0}\right)$ et où l'on ne considère que le bilan global du drainage - e'est-à-dire le volume d'eau total écoulé entre le début du drainage et l'équilibre.

Pour le drainage d'une nappe de surface, on peut considérer deux cas, selon que le rabattement $Z$ est supérieur ou inférieur à la hauteur de la frange capillaire. Si $Z \leqslant z_{e}$, le volume d'eau drainé est nul. Si $Z>z_{e}, V_{\infty}$ est proportionnel à $Z-z_{\epsilon}$.

Dans le cas d'une nappe située à une profondeur initiale $\mathrm{H}$, on distinguera également deux cas :

si $0<\mathrm{H} \leqslant z_{\varepsilon}, V_{\infty}$ est proportionnel à :

$$
\mathrm{Z}-\left(z_{e}-\mathrm{H}\right) \text {; }
$$

- si $\mathrm{H}>z_{c}$, ce volume est proportionnel à $Z$.

L'équation générale de l'écoulement (7) s'écrit plus communément sous la forme:

$$
\frac{\partial \theta}{\partial t}=\frac{\partial}{\partial z}\left(\mathrm{D} \frac{\partial \theta}{\partial z}\right)+\frac{\partial k}{\partial z}
$$

où $\mathrm{D}=k(\partial \psi / \partial \theta)$ est le coefficient de diffusitivité (Philip, 1958). L'artifice de calcul provoqué par l'introduction de D- qui n'a aucune signification physique, et qui ne doit surtout pas donner l'impression que les écoulements d'eau en milieu poreux non saturé se ramènent à des problèmes de diffusion moléculaire - revient en fait à faire passer les équations de l'écoulement dans une classe plus générale: celle des équations de transport avec un coefficient de diffusion quelconque.

L'utilisation de (1) et (1') masque done en fait trois réalités :

a) l'existence d'une frange capillaire à teneur en eau constante;

b) l'existence au-dessus de cette frange d'une zone à teneur en eau variable, où le potentiel spécifique à une teneux en eau donné $\theta$ est lié à cette valeur par la courbe de succion;

c) le fait que l'eau située dans cette zone non saturée ne s'écoule pas instantanément, mais que les variations de teneurs en eau $\theta(z, t)$, fonctions des caractéristiques $k$ et $\psi$ du matériau et des conditions initiales et aux limites du problème considéré, sont obtenues en résolvant l'équation (7) ou (8).

Cependant, la solution de cette équation n'est possible qu'en utilisant des méthodes numériques relativement complexes, sur des calculateurs à grande capacité. De plus, cette équation repose sur l'hypothèse de validité de la loi de Darcy généralisée, hypothèse qui n'a été que très rarement vérifiée. Quand bien même cette hypothèse serait valable, il faut en outre avoir les lois $\psi(\theta)$ et $k(\theta)$ pour résoudre (7), mais la seconde est généralement inconnue. Enfin, le contrôle des solutions $\theta(z, t)$ nécessite la mesure rapide et précise des teneurs en eau durant l'écoulement, mesure qui ne pent s'effectuer valablement qu'avec des techniques d'absorption - ou de thermalisation - de rayonnements nucléaires, donc avec un matériel relativement élaboré.
Les difficultés rencontrées à ces différents niveaux peuvent expliquer l'utilisation abusive des formules (1) et (1') et le nombre restreint d'études effectuées sur ce problème de drainage en tenant compte de l'écoulement dans la zone non saturée.

Les premiers résultats analysant l'erreur introduite dans les calculs de drainage de nappe en adoptant l'hypothèse d'une porosité effective constante, sont dus à Luthin-Worstell (1957). A partir d'une cuve à sable, et en mesurant l'évolution des pressions capillaires dans le sol et le profil de la nappe durant le drainage, ces auteurs montrent que, pour un sol à granulométrie étroite (sable bien calibré), l'hypothèse $S=\varepsilon$, revient en fait à substituer le niveau de la frange capillaire au niveau de la nappe.

D'autres analyses qualitatives ont ensuite été effectuées par Childs (1960), Childs-Poulovassilis (1962), Youngs (1963) et Degallier (1965).

Les premiers essais de résolution de l'équation (8) -- appliquée au problème du drainage des sols - sont dus à Day et Luthin (1956). Mais les résultats, obtenus sous la forme : variation de pression totale en fonction du temps, sont peu facilement exploitables. Il semble que le pas d'itération soit trop grand, et la méthode de calcul choisie est longue et laborieuse. Des essais expérimentaux effectués simultanément avec des tensiomètres dans une colonne de sol ne sont guère exploitables non plus, car tout le début du drainage n'est pas exploré, du fait de l'inertie des tensiomètres. Ce n'est qu'à partir de 1965 qu'une méthode approchée satisfaisante de résolution numérique de l'équation (8) a pu être obtenue (Remson, 1965; Liakopoulos, 1965; Watson, 1967; Remson, 1967), bien qu'il faille noter que seul Watson apporte des résultats expérimentaux intéressants à l'appui des résultats théoriques.

Entre temps, un certain nombre d'essais d'analyse approximative de la physique du drainage ont été présentés en s'appuyant, soit sur une analogie avec l'écoulement de Poisenille dans un milieu capillaire (Youngs, 1960), soit sur une hypothèse de linéarité de la loi $\mathrm{D}(\theta)$ (Gardner, 1962; Fujioka, 1964).

Les premiers essais expérimentaux réellement exploitables remontent à 1960 (Taylor, 1960), mais ces résultats ne permettent d'apprécier que la valeur dı coefficient d'emmagasinement $S$ à l'équilibre, et on ne peut en déduire ni l'évolution des profils hydriques durant le drainage, ni la valeur de $\mathrm{S}$ en régime transitoire. Les seules données utilisables sont en fait celles de Prill-Johnson (1965) et de Watson (1966). Les premiers mesurent l'évolution des pressions capillaires durant le drainage d'une nappe de surface dans des colonnes de sables et billes de verre en utilisant des tensiomètres; le dernier obtient directement les profils hydriques par absorption de rayons $\gamma$. Mais Watson n'étudie que le début du drainage, alors que les méthodes expérimentales utilisées par Prill-Johnson permettent seulement d'analyser l'écoulement au bout d'un temps relativement long (au moins une heure), et jusqu'à la fin du drainage (inertie des tensiomètres).

Signalons enfin la parution toute récente d'une étude de Jensen (1967) sur un sujet très proche de celui que nous exposons ici, et se basant sur les mêmes méthodes de mesure. 
A partir d'essais de drainage effectués sur des colonnes verticales de sable, nous avons essayé de rechercher la loi de variation du coefficient d'emmagasinement en fonction du temps, du rabattement et de la profondeur de la nappe, et nous analysons l'erreur résultant de l'utilisation de l'hypothèse (1') sur des modèles d'écoulements couramment utilisés. Puis, à partir de l'évolution des teneurs en eau pendant le drainage, évolution obtenue par absorption de rayons $\gamma$, nous donnons une méthode de détermination de la conductivité hydraulique $k(\theta)$, ce qui nous conduit à vérifier, pour le type de sol considéré, la validité de la loi de Darcy généralisée $(6 b)$.

\section{II. - Méthode expérimentale}

\section{Caractéristiques et mise en place du sol.}

Les essais ont été effectués sur la portion inférieure à $2 \mathrm{~mm}$ d'un sable de rivière. On trouvera figure 2 la courbe granulométrique du matériau, ainsi que la courbe de pression capillaire en drainage (à l'équilibre) obtenue séparément par un essai classique de plaque de succion sur un échantillon de même sol, compacté à la même masse volumique que la colonne de sable.

Le sable est compacté à une teneur en eau initiale $\theta_{i}=0,025 \mathrm{~cm}^{3} / \mathrm{cm}^{3}$ dans une colonne d'Altuglass de $180 \mathrm{~cm}$ de haut et de $9,02 \mathrm{~cm}$ de diamètre intérieur. Pour obtenir un compactage uniforme, le sol est versé par petites quantités, puis chaque couche est vibrée extérieurement avec un maillet de bois sous une énergie constante. Lorsque la colonne entière est remplie, une prospection aux rayons $\gamma$ permet de vérifier l'homogénéité en masse volumique. Si la largeur de bande de dispersion des valeurs ponctuelles de la masse volumique autour de la valeur moyenne est supérieure $\pm 2 \%$, la colonne est détruite et reconstituée. La masse volumique moyenne de la colonne de sol utilisé vaut:

$$
\rho_{l}=1,61 \pm 0,025 \mathrm{~g} / \mathrm{cm}^{3}
$$

La base de la colonne est fixée sur un filtre de $10 \mathrm{~cm}$ de gravier, relié à une burette de Mariotte. Avant le début des essais, la burette de Mariotte est remontée progressivement jusqu'à ce que la surface libre de la nappe arrive à $5 \mathrm{~mm}$ au-dessus de la surface du sol, et un écoulement permanent est maintenu pour mesurer la perméabilité du sol sous la nappe.

Cette perméabilité vaut : $K_{0}=1,09 \mathrm{~cm} / \mathrm{mn}$ pour une teneur en eau moyenne (obtenue par prospection de rayons $\gamma$ ):

$$
\theta_{0}=0,342 \mathrm{~cm}^{3} / \mathrm{cm}^{3}
$$

Il faut noter que $\theta_{0}$ est sensiblement inférieur à la porosité du sol, eslimée à partir du poids spécifique et de la masse volumique du sol, qui vaut approximativement :

$$
n \simeq 0,41 \mathrm{~cm}^{3} / \mathrm{cm}^{3}
$$

la différence $\theta_{0}-n$ représente le volume d'air emprisonné dans le sol durant la remontée de la nappe. Cependant, en accord avec les résultats de Croney-Coleman (1958), Topp (1966), cette quantité est remarquablement constante durant les cycles de drainage successifs sur la même colonne de sol.

Lors d'un premier drainage, la nappe est instantanément rabattue de $Z$ (fig. 1 ). Les teneurs en eau sont mesurées selon la méthode décrite au paragraphe suivant, et le volume d'eau drainé est mesuré par la burette de Mariotte. A la fin de l'essai, le niveau de la nappe est soit remonté au sommet de la colonne pour un nouvel essai de drainage -- à partir de la même teneur en eau pour un sol de même masse volumique, avec un rabattement $Z^{\prime}-$, soit rabattu d'une nouvelle quantité $\Delta Z$. Dans le premier cas, on obtient les caractéristiques du drainage d'une nappe de surface, dans le second, celles du drainage d'une nappe en profondeur à la cote initiale $z=\mathrm{L}-Z$.

\section{Détermination des teneurs en eau.}

Les teneurs en eau sont déterminées par absorption de rayons $\gamma$ selon la méthode expérimentale décrite par Wack (1961), Benblidia (1963), Davidson (1963), Reginato-Van Bavel (1964) et Nielsen-Vachaud (1964).

La colonne de sol est fixée contre un montant verlical qui sert de guide à une plateforme encadrant la colonne (photo 1). Sur cette plateforme, sont montés de part et d'autre de la colonne une source de 200 millicuries de ${ }^{137} \mathrm{Cs}$ et un détecteur à photomultiplicateur avec cristal de $\mathrm{INa}$ de 1,5 pouce. Les rayons $\gamma$ émis par la source sont collimatés en un faisceau de $3 \mathrm{~mm}$ de diamètre par un bloc de plomb de $50 \mathrm{~mm}$ d'épaisseur, et sont astreints à passer dans un plan diamétral vertical de la colonne. La plateforme est mue par un moteur électrique à mouvement réversible.

Les valeurs obtenues selon les procédés classiques (Davidson, 1963) sont respectivement :

$$
\begin{aligned}
& \mu_{w}=0,08297 \pm 0,00016 \mathrm{~cm}^{2} / g \\
& \mu_{s}=0,07739 \pm 0,00075 \mathrm{~cm}^{2} / \mathrm{g}
\end{aligned}
$$

Les variations de teneur en eau sont données par la formule classique :

$$
\ln \frac{\mathrm{N}_{0}}{\mathrm{~N}}=x\left(\rho_{w} \mu_{u v} \theta+\rho_{t} \mu_{k}\right)
$$

Pour un temps de comptage de $50 \mathrm{~s}$, une teneur en eau $\theta$ de $30 \%$ et un degré de certitude de $95 \%$, on a dans ces conditions :

$$
\frac{\Delta \theta}{\theta} \leqslant \pm 2,5.10^{-2}
$$

Les variations de comptage dues aux fluctuations de l'appareillage de mesure sont prises en compte en contròlant, avant et après les mesures, le taux de comptage à travers un écran standard.

Afin d'obtenir l'évolution des teneurs en eau à différents niveaux successifs de la colonne, il est nécessaire de procéder à un déplacement par paliers de la plateforme de mesure. Cette plateforme est initialement positionnée pour que le faisceau 


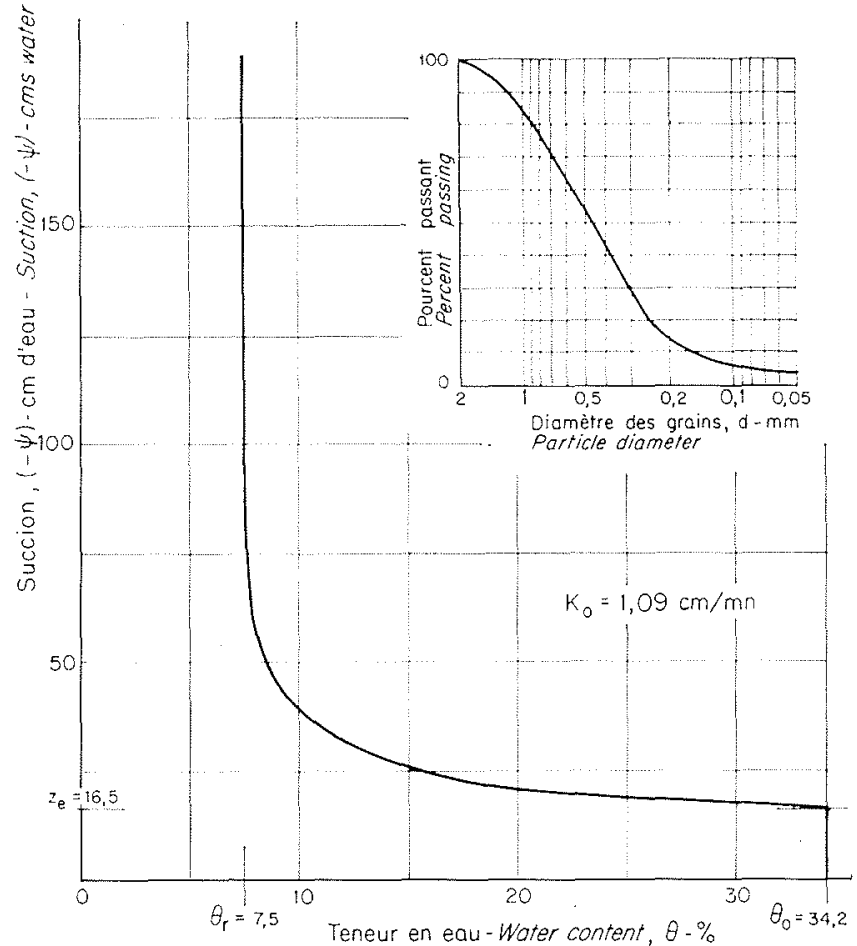

2/ Courbe de suceion et distribution granulométrique pour le sable de rivière utilisé.

Suction curve and grain size characteristics for the river sund used.

de rayons $\gamma$ soit situé dans un plan voisin du sommel de la colonne. Tant que la teneur en eau de la section de mesure reste fixe, les taux de comptage sont constants, puis ils varient brusquement au passage du front correspondant à la teneur en eau $\theta_{0}$. La plateforme est alors descendue pour étudier l'évolution des teneurs en eau dans une section de mesure initiale, etc.

L'écoulement étant très rapide au début (le front de teneur en eau $\theta_{0}$ passant de la cote $180 \mathrm{~cm}$ à la cote $60 \mathrm{~cm}$ durant les dix premières minutes), et la fréquence des mesures étant limitée par la durée des complages, qui doit être d'au moins $20 \mathrm{~s}$ pour avoir une précision suffisante, le nombre de sections explorées est assez restreint durant cette période : elles sont espacées d'environ $20 \mathrm{~cm}$. Les variations de taux de comptage sont alors directement enregistrées graphiquement.

Au fur et à mesure que le drainage progresse, l'écoulement devient beaucoup plus lent et il devient possible d'augmenter le nombre et la durée des mesures. Les temps de comptage moyens passent alors à $50 \mathrm{~s}$, avec impression sur enregistreur digital.

On trouvera figure 3 les résultats des mesures obtenus par cette méthode pour un rabattement de $180 \mathrm{~cm}$. Chaque point représente une prospection dans la section considérée.

Par changement de paramètre on peut facilement, à partir de la figure 3 , obtenir la variation des pro-

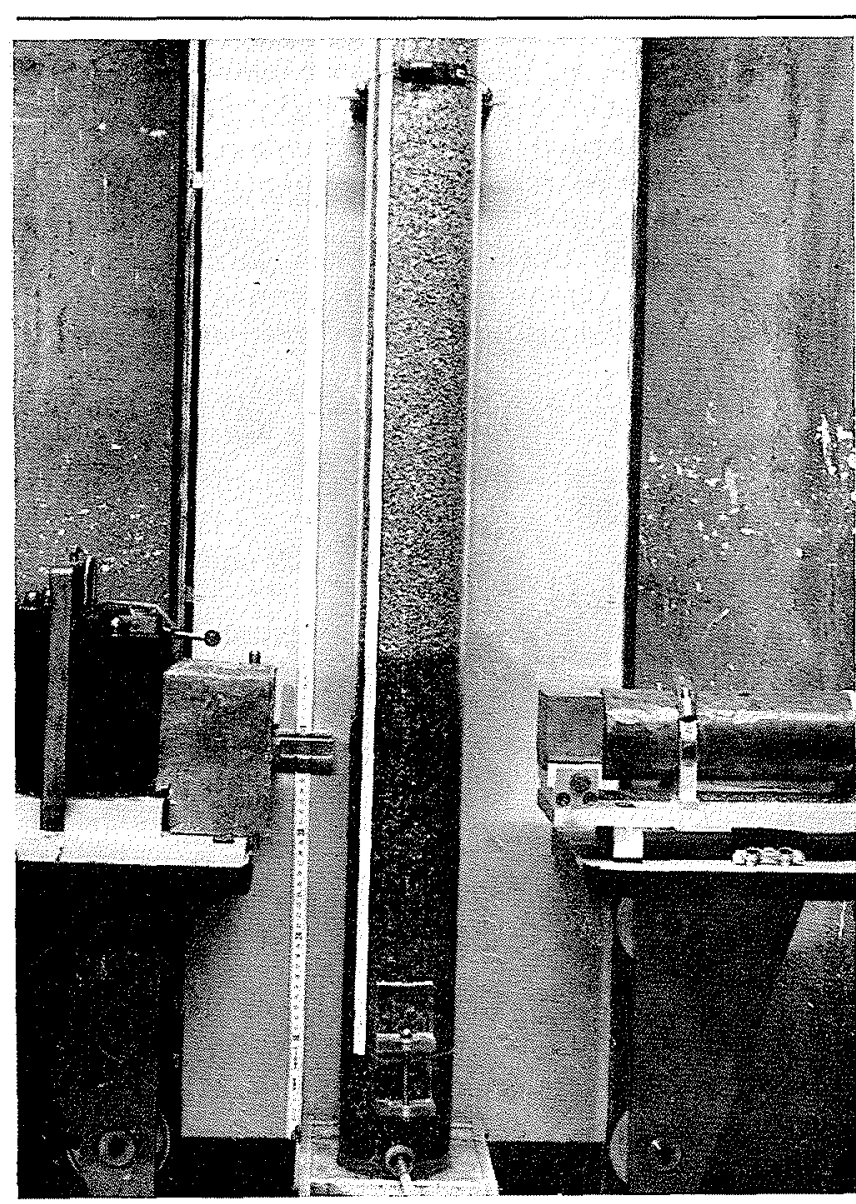

Photo $1 /$

Essai de drainage. Plateforme de mesures pour colonnes verticales.

Irainage test. Vertial column measurement platform.

fils hydriques dans la colonne durant le drainage. On trouvera ainsi figure $4 a$ les profils hydriques obtenus pour l'essai de drainage défini par $H=0, Z=180 \mathrm{~cm}$, dont les résultats directs sont donnés figure 3 .

La figure $4 b$ représente les résultats de deux essais de drainage successifs correspondant aux conditions suivantes:

$$
\begin{aligned}
& \mathrm{H}=0, \quad Z=103 \mathrm{~cm} \\
& \mathrm{H}=103, \quad Z=77 \mathrm{~cm}
\end{aligned}
$$

Le second essai a été effectué $22 \mathrm{~h}$ après le début du premier, et avant que la courbe d'équilibre (en pointillés sur la figure $4 b$ correspondant à cet essai) n'ait été atteinte.

L'étude détaillée de l'évolution de $\theta(z, t)$ fait l'objet d'un chapitre spécial.

\section{III. - Valeur du volume d'eau drainé et du coefficient d'emmagasinement Etude des modèles}

Les courbes reportées figure 5 représentent la variation du volume d'eau écoulé à la base de la colonne en fonction du temps et du rabattement pour cing essais effectués à partir d'une nappe affleurant initialement la surface du sol $(\mathrm{H}=\mathbf{0})$. 
Les points reportés sur ces courbes correspondent aux valeurs de $V_{w}$ obtenues soit par lecture de la burette de Mariotte reliée à la base de la colonne, soit par intégration des profils hydriques $\theta(z, t)$.

Ces essais sont relatifs à des rabattements de 49 , $77,103,143$ et $180 \mathrm{~cm}$, pour une colonne de sol de $180 \mathrm{~cm}$ de long. Dans chaque cas, la courbe $V_{w}(t)$ tend vers une valeur limite $V_{\infty}$ donnée par intégration du profil d'équilibre $\psi(\theta)$ correspondant :

$$
\mathrm{V}_{\infty}=\mathrm{A} \int_{0}^{\mathrm{Z}}\left(\theta_{0}-\theta\right) d \psi
$$

Pour les essais considérés, $V_{\infty}$ vaut respectivement $410,890,1310,2010$ et $2630 \mathrm{~cm}^{3}(\mathrm{~A}=63,93$ $\mathrm{cm}^{2}$ ).

Pour étudier l'influence de la frange capillaire et de la zone non saturée existant initialement au-dessus de cette frange sur les valeurs des volumes d'eau drainés, il est intéressant de comparer les deux essais de drainage reportés figure $4 b$. Afin de simplifier l'exposé, adoptons les dénominations suivantes :

- drainage $\quad$ I. ........ H $=0 \quad \mathrm{Z}=180 \mathrm{~cm}$

- drainage II. ....... $\mathrm{H}=0 \quad \mathrm{Z}=103 \mathrm{~cm}$

- drainage III........ H $\mathrm{H}=103 \mathrm{Z}=77 \mathrm{~cm}$

Compte tenu de l'effet de la frange capillaire, on pourrait, dans un premier stade, essayer de compa- rer le drainage III à un drainage de nappe de surface effectué avec un rabattement :

$$
Z *=Z+z_{e}=93,5 \mathrm{~cm}
$$

donc avec un essai sensiblement équivalent à l'essai II, en tenant compte de l'influence de la zone non saturée. Cette comparaison ne peut cependant avoir de valeur qu'en terme de bilan global. En effet, il faut remarquer que, dans le cas du drainage III, la position finale de la nappe correspond an niveau de la base de la colonne, alors que, dans l'essai II, il existe une distance de $77 \mathrm{~cm}$ entre la nappe (position finale) et la base de la colonne. Si bien qu'avec des rabattements équivalents, les gradients initiaux, obtenus en considérant le milieu comme saturé durant les premières secondes du drainage, seront assez différents pour les cas II et III.

Les valeurs des volumes drainés et les débits correspondant aux trois essais I, II, III, sont reportées figure 6 pour les cent premières minutes de chaque essai. Les courbes $V_{w}(t)$ relatives aux essais I et II sont naturellement les mêmes que celles données figure 5. Les cercles entourant ces courbes correspondent aux valeurs des volumes obtenues par intégration des profils hydriques, reportées figure 4, aux temps correspondants. Ces résultats sont également

\begin{tabular}{|c|c|c|c|c|c|c|c|c|c|c|c|c|}
\hline \multirow{3}{*}{$t$} & \multicolumn{8}{|c|}{$\mathrm{H}=0$} & \multicolumn{4}{|c|}{$\mathrm{H}=103 \mathrm{~cm}$} \\
\hline & \multicolumn{4}{|c|}{$Z=180 \mathrm{~cm}$} & \multicolumn{4}{|c|}{$Z=103 \mathrm{~cm}$} & \multicolumn{4}{|c|}{$Z=77 \mathrm{~cm}$} \\
\hline & $\mathrm{V}_{w}$ & $q$ & $\mathrm{~V}_{10} / \mathrm{V}_{\infty}$ & $\tau$ & $V_{n}$ & $q$ & $V_{w} / V_{x}$ & $\tau$ & $V_{w}$ & $q$ & $V_{u} / V_{\infty}$ & $\tau$ \\
\hline 0 & 0 & 0,990 & 0 & 0 & 0 & 0.715 & 0 & 0 & 0 & 0.90 & 0 & 0 \\
\hline 2 & 120 & 0,95 & 0,046 & 0,048 & 70 & 0,67 & 0.054 & 0,070 & 110 & 0.82 & 0.086 & 0.086 \\
\hline 5 & 305 & 0,895 & 0,116 & 0,120 & 210 & 0,55 & 0.161 & 0.175 & 290 & 0.68 & 0.216 & 0.22 \\
\hline 10 & 565 & 0,77 & 0,215 & 0,240 & 415 & 0,44 & 0.317 & 0.35 & 495 & 0.52 & 0.369 & 0.43 \\
\hline 15 & 800 & 0,64 & 0,304 & 0,361 & 540 & 0,32 & 0.412 & 0.524 & 650 & 0.36 & 0.484 & 0.65 \\
\hline 20 & 980 & 0,51 & 0,373 & 0,481 & 620 & 0,24 & 0.473 & 0.700 & 750 & 0.26 & 0.558 & 0.86 \\
\hline 30 & 1240 & 0,34 & 0,471 & 0,722 & 735 & 0,15 & 0.561 & 1.05 & 870 & 0.14 & 0.647 & 1.30 \\
\hline 45 & 1500 & 0,21 & 0,571 & 1,085 & 855 & 0,09 & 0.653 & 1.57 & 960 & 0.09 & 0.715 & 1.95 \\
\hline 60 & 1635 & 0,135 & 0,622 & 1,444 & 930 & 0,059 & 0.710 & 2.10 & 1015 & 0.058 & 0.756 & 2.60 \\
\hline 120 & 1940 & 0,058 & 0,738 & 2,888 & 1080 & 0,021 & 0.825 & 4.20 & 1120 & 0.026 & 0.834 & 5.20 \\
\hline 240 & 2150 & 0,020 & 0,817 & 5,78 & 1170 & $7.10^{-3}$ & 0.894 & 8.38 & 1210 & 0.010 & 0.900 & 10.50 \\
\hline 360 & 2230 & 0.010 & 0,848 & 8,664 & 1200 & $4,2.10-3$ & 0.916 & 12.60 & 1250 & $5.10-3$ & 0.930 & - \\
\hline 600 & 2340 & $4,5.10-3$ & 0,889 & 14,45 & 1260 & $2.10-3$ & 0.961 & & 1275 & $3: 10-3$ & 0.947 & - \\
\hline 1440 & 2460 & $1,2.10-3$ & 0,935 & & 1280 & $6 \quad .10^{-4}$ & 0.977 & & 1320 & - & 0.982 & $\ldots$ \\
\hline 5000 & 2560 & $2.10-4$ & 0,973 & & 1300 & - & 0.987 & & 1340 & $\ldots$ & 0.996 & \\
\hline $10^{4}$ & 2590 & - & 0,985 & & & & & & & & & \\
\hline Equil. & 2630 & & & & 1310 & & & & 1345 & & & \\
\hline
\end{tabular}
reportés en clair dans le tableau 1 .

Unités : $t, \mathrm{mn} ; \mathrm{V}_{w}, \mathrm{~cm}^{\mathrm{a}} ; q, \mathrm{~cm} / \mathrm{mn}$. 


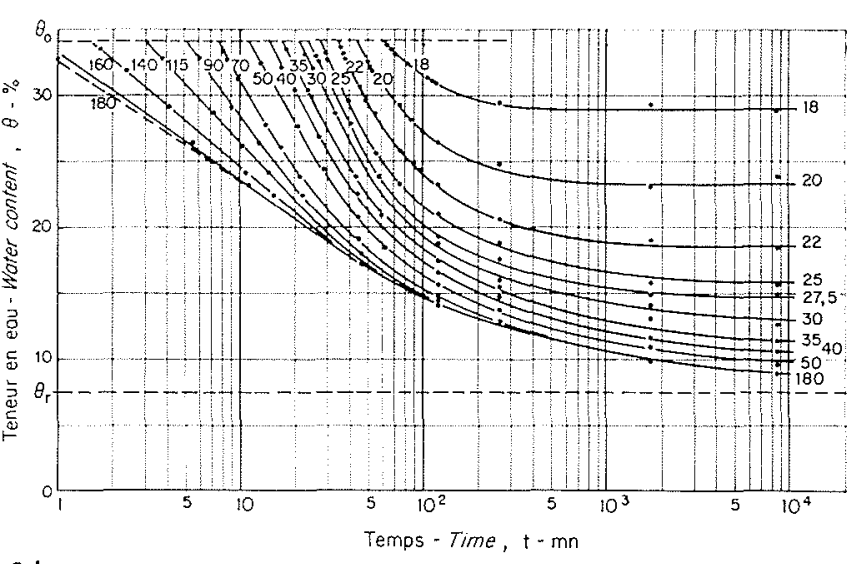

3/ Variation des teneurs en eau durant le drainage. $\mathrm{H}=0$, $Z=180 \mathrm{~cm}$. Paramètre $=$ cote $a u-d e s s u s ~ d u$ fond de la colonne.

Water content variation during drainage. $\quad H=0, Z=$ $180 \mathrm{~cm}$. Parameter: height above column bottom.

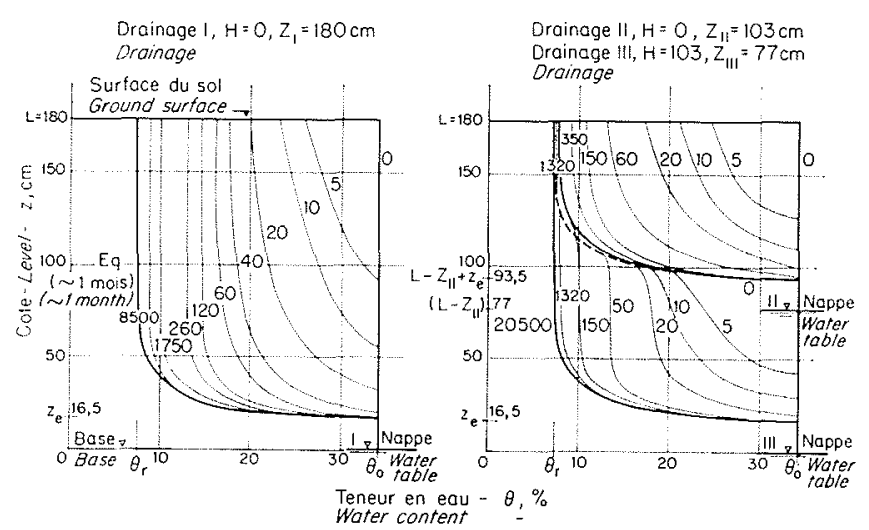

4/ Répartition des profils hydriques pendant deux essais de drainage de surface et un essai de profondeur. Paramètre $=$ temps $t$, en minutes, à partir du début $\mathrm{du}$ drainage.

Water profile variation during two surface drainage tests and one deep drainage test. Parameter: Time t from start of drainage, in minutes.

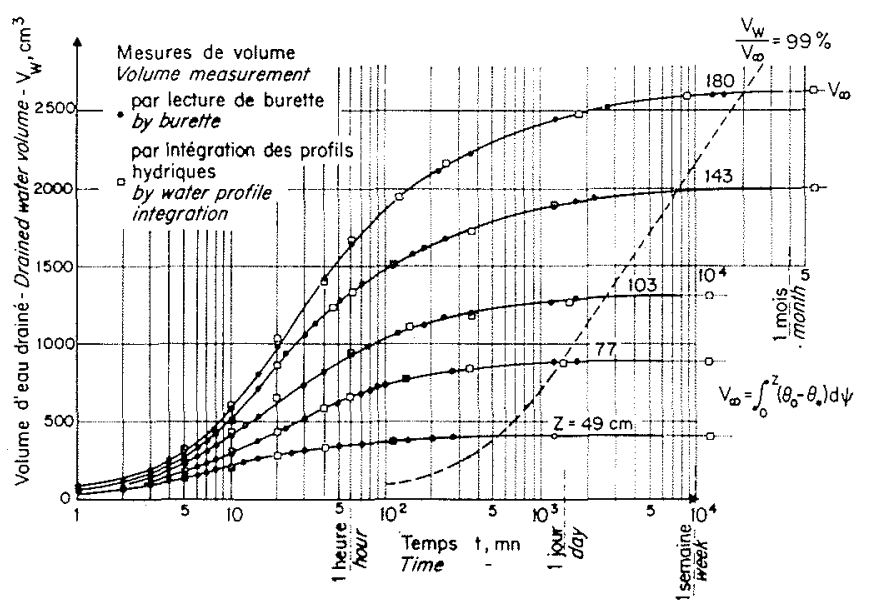

5/ Variation des volumes d'eau écoulés par la base de la colonne pendant cing essais de drainage de surface. Paramètre $=$ rabattement $Z$ en centimètres.

Variation in volumes of water flowing out at the bottom of the column during five surface drainage tests. Parameter: drawdown $Z$ in $\mathrm{cms}$.

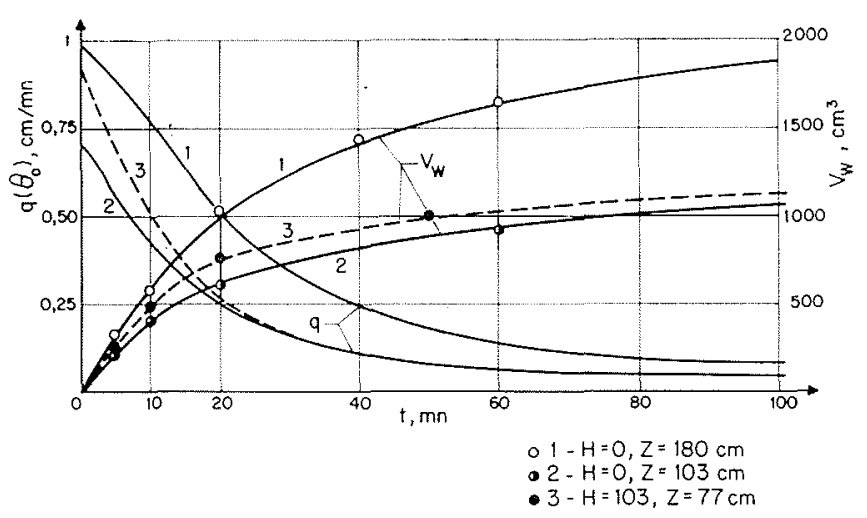

6/ Variation des débits unitaires et des volumes drainés pendant les 100 premières minutes pour les trois essais reportés figure 5 .

Unit discharge and llained volume variation during the first 100 minutes for the three tests shown in Figure 5.
On voit que l'influence des gradients initiaux est importante sur la dynamique des écoulements. Alors que les valeurs du volume total drainé $V_{\infty}$ sont pratiquement identiques pour les essais II et III, les valeurs instantanées des volumes drainés $V_{w}$ sont relativement différentes, surtout durant les vingt premières minutes de l'écoulement.

Cette influence apparaît encore plus nettement en termes de débil : les débits deviennent identiques pour $t>20 \mathrm{mn}$ mais la valeur $d u$ débit initial $q_{0}$ est nettement supérieure pour l'essai III à la valeur obtenue pour l'essai II. Les valeurs des débits $q$ sont obtenues directement en prenant la pente des courbes $\mathrm{V}_{w}(\boldsymbol{t})$.

Mais il est bien évident, si l'on se reporte à la figure 5, qu'une erreur considérable résulterait de l'estimation des volumes drainés, et des débits, correspondant à l'essai III à partir des valeurs obtenues pour l'essai de surface $\mathrm{H}=0, Z=77 \mathrm{~cm}$ effectué avec le même rabattement que l'essai III. On négligerait en effet, dans un tel cas, non seulement l'influence de la valeur du gradient initial sur la dynamique de l'écoulement, mais aussi celle de la frange capillaire et de la zone non saturée.

On trouvera également tableau 1 les valeurs de:

$$
\begin{aligned}
\frac{V_{w}}{V_{\infty}} & =\text { volume drainé réduit } \\
\tau & =\frac{q_{0} \cdot A}{V_{\infty}} t=\text { temps réduit }
\end{aligned}
$$

où $q_{0}$, débit initial, correspond à la pente de la courbe $V_{w}(t)$ à l'origine.

Les valeurs de $V_{w} / V_{\infty}$ et $\tau$ sont introduites pour tester, à partir des résultats globaux obtenus ciavant, la valeur des modèles couramment utilisés pour simuler les essais de drainage.

Ces modèles sont de deux types, les deux étant d'ailleurs basés sur l'hypothèse $S=\varepsilon$.

Le premier - modèle gravitaire — revient à supposer que la surface de la nappe est la limite entre la zone saturée et le sol sec. On néglige donc l'influence de la frange capillaire et de la zone non saturée. C'est le modèle implicitement adopté dans la formulation de $(1)$ et $\left(1^{\prime}\right)$. 


\section{A. vachaud}

Lots dim rabaltement $Z$, tout l'écoulement a Gono lieu dans la zone saturée, avec un débit unithire constant :

$$
q=-\mathrm{K}_{0}
$$

Le volume d'eau drainé au temps $t$ vaut:

$$
\mathrm{V}_{w}=\mathrm{A} \cdot \varepsilon \cdot q_{0} t=\mathrm{A} \cdot \varepsilon \cdot \mathrm{K}_{0} \cdot t
$$

Le volume total drainable est donné par (1') :

$$
\mathrm{V}_{\infty}=\varepsilon \cdot \mathrm{A} . \mathrm{Z}
$$

Le drainage se termine donc au temps $t^{\prime}$ tel que :

$$
t^{\prime}=\frac{Z}{\mathrm{~K}_{0}}
$$

Le second modèle - modèle capillaire (Youngs, 1960), prend en compte l'influence de la frange capillaire. Il doit donc mieux s'adapter aux cas réels que le modèle précédent. Il est basé sur l'analogie entre l'écoulement en milieu poreux et l'écoulement dans un faisceau de capillaires de diamètre uniforme, l'écoulement dans chaque capillaire répondant à la loi de Poiseuille, et le débit s'arrètant lorsque, dans chaque tube, la cote de l'eau audessus de la surface de la nappe est égale à la hauteur de remontée capillaire.

Soient :

$r$ le diamètre de chaque tube;

$z$ la cote au-dessus de la nappe à un instant $t$;

$h_{0}$ la hauteur de remontée capillaire (loi de Jurin).

Au temps $t$, le débit est donné pour chaque tube par :

$$
-\pi r^{2} \frac{d z}{d t}=\frac{\rho_{u} g}{8 \eta} \pi r^{4} \frac{z-h_{0}}{z}
$$

En comparant avec la loi de Darcy, on voit que :

$$
\mathrm{K}_{0} \sim \frac{\mathrm{p}_{w} g}{8 \eta} r^{2}
$$

où $\eta$ est la viscosité dynamique de l'eau.

Si l'on considère un faisceau de $n$ tubes et si l'on introduit les valeurs suivantes:

$$
\begin{aligned}
\mathrm{V}_{w}=n \pi r^{2}(Z-z) & =\text { volume de liquide } \\
& \text { drainé au temps } t, \\
\mathrm{~V}_{\infty}=n \pi r^{2}\left(Z-h_{0}\right) & =\text { volume total drainable, } \\
q_{0}=\frac{\rho_{m} g}{8 \eta} r^{2} \frac{Z-h_{0}}{Z} & =\text { débit unitaire initial, } \\
\tau & =\frac{n \pi r^{2} q_{0}}{\mathrm{~V}_{\infty}} t=\frac{\mathrm{K}_{0}}{\mathrm{Z}} t=\text { temps réduit; }
\end{aligned}
$$

on peut mettre l'expression ci-dessus, après développement en série et en ne conservant que le terme du premier ordre, sous une forme de variables réduites :

$$
\frac{V_{w}}{V_{\infty}}=1-e^{-\tau}
$$

On voit facilement qu'en variables réduites, l'expression (12), établie pour le modèle gravitaire, se ramène à :

$$
\frac{V_{w}}{V_{\infty}}=\tau
$$

Pour étudier la validité de ces deux modèles à simuler, en terme de bilan, le drainage des sols, nous avons reporté figure 7 les résultats expérimentaux de drainage, en utilisant les mêmes variables réduites. Pour passer du modèle capillaire au sol, on voit que l'analogie ne tient compte que de la hauteur de la frange capillaire. Il suffit donc de remplacer $h_{0}$ par $z_{e} \cdot V_{w}$, et $V_{\infty}$ et $q_{0}$ intervenant dans l'expression de $\tau$, sont directement obtenus à partir des mesures de volumes. La surface de la colonne de sol vaut : $A=63,93 \mathrm{~cm}^{2}$. Les points reportés figure 7 sont relatifs aux cinq essais de drainage effectués avec $\mathrm{H}=0$ (fig. 5) et à l'essai III.

On voit d'après la figure 7 que la courbe expérimentale, correspondant à un sol donné, est une courbe unique. Au début du drainage, cette courbe coïncide avec celle résultant du modèle capillaire, mais assez rapidement le modèle capillaire conduit à des valeur's de volume drainé bien supérieures à celles obtenues expérimentalement. Il suffit d'ailleurs de se référer aux résultats reportés figure 4 pour constater que le drainage n'est pas terminé lorsque le front de teneur en eau $\theta_{0}$ atteint la cote $z_{e}$, comme l'impliquerait la théorie du modèle capillaire.

Il est d'ailleurs normal que ce modèle ne puisse décrire le drainage, puisqu'il ne tient compte que de la frange capillaire, revenant en fait à substituer à la distribution de teneur en eau donnée par la courbe $\psi(\theta)$ une distribution correspondant à :

$$
\begin{array}{ll}
\theta=\theta_{0} & z \leqslant z_{e} \\
\theta=0 & z>z_{e}
\end{array}
$$

Afin d'obtenir un modèle plus apte à représenter la physique du drainage, nous proposons de considérer une famille de $n_{i}$ tubes de rayon $r_{i}$ choisis de façon à approcher au mieux la courbe d'équilibre en drainage.

Considérons (tabl. 3) une courbe d'équilibre en drainage après un rabattement $Z$ de la nappe, et soient :

$\theta_{1}$ la valeur de la teneur en eau à la surface du sol;

$\theta_{0}$ la valeur de la teneur en eau au niveau de la nappe;

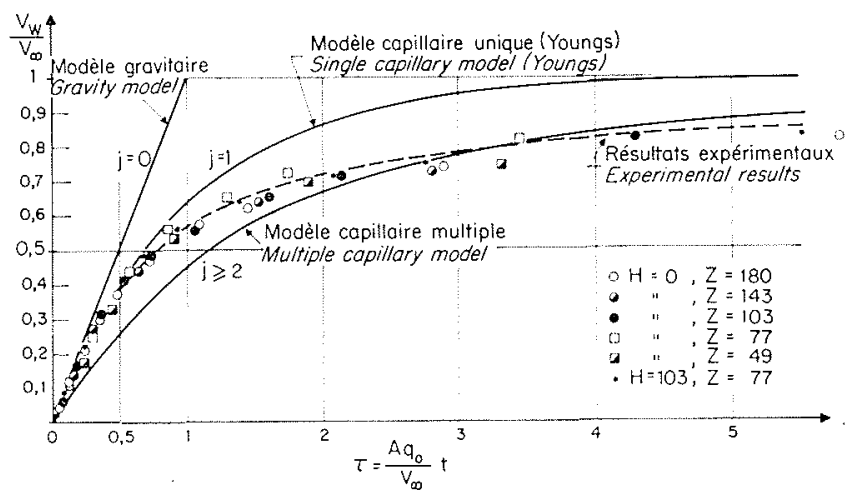

7/ Analyse de la validité des modèles gravilaires et capillaires. Influence du nombre $j$ de familles de tubes.

Analysis of validity of gravity and capillary models. Effect of the number of tube families $\mathrm{j}$. 
$z_{e}$ la hauteur de la frange capillaire.

Prenons $j$ familles de tubes de rayon $r_{i}$ tels que:

$$
\sum_{i=1}^{j} n_{i} \pi r_{i}^{2} h_{i}=\int_{\theta_{1}}^{\theta_{0}} \psi d \theta
$$

où $n_{i}$ est la densité de tubes $r_{i}$ par surface unitaire, et $h_{i}$ est la hauteur de remontée capillaire dans chaque tube. Fixons-nous entre les $n_{i}$ et les $r_{i}$ une relation de la forme:

$$
n_{1} r_{1}^{2}=n_{2} r_{2}^{2} \ldots=n_{j} r_{j}^{2}
$$

Ce qui revient à considérer que les sections d'écoulement sont égales pour chaque famille de tubes, ou à découper la zone de teneur en eau $\theta_{0}-\theta_{1}$ en $j \Delta \theta$ segments identiques.

Choisissons enfin pour la première famille de tubes un rayon $r_{1}$ correspondant à une remontée capillaire $h_{1}$ telle que :

$$
h_{1}=z_{\varepsilon}
$$

Le choix des $h_{i}$ est donc fixé facilement, de facon à approcher au mieux, pour chaque segment $\Delta \theta_{i}$, l'aire limitée par la courbe d'équilibre, les valeurs de $\theta_{i-1}$ et $\theta_{i}$ et l'axe $\psi=0$.

Pour chaque faisceau de tubes, il est possible d'écrire indépendamment la loi de Poiseuille comme dans le cas d'un faisceau de tubes uniformes, ce qui conduit à $j$ relations de la forme :

$$
\frac{V_{w_{t}}}{V_{\infty_{i}}}=1-e^{-\tau_{i}}
$$

avec, dans chaque cas :

$$
\tau_{i}=\frac{\alpha r_{i}^{2}}{\mathrm{Z}} t \quad \alpha=\frac{\rho_{w} g}{8 \eta}
$$

Si l'on se réfère aux tubes de rayon $r_{1}$, correspondant à $z_{e}$, on peut définir une nouvelle variable adimensionnelle :

$$
\xi_{i}=\frac{z_{6}}{h_{i}}
$$

Soit $\tau$ le temps réduit de référence fixé par la famille de tubes $r_{1}$, on a alors entre les $\tau_{i}$ une relation du type :

$$
\tau_{i}=\tau \xi_{i}^{2}
$$

d'où l'on déduit :

$$
\frac{\mathrm{V}_{w}}{\mathrm{~V}_{\infty}}=\frac{\sum_{i=1}^{j}\left[\left(\mathrm{Z}-\xi_{i} z_{c}\right)\left(1-e^{-\tau \xi^{2}}\right)\right]}{j Z-z_{e} \sum_{i=1}^{j} \xi_{i}}
$$

Afin de tester la convergence des séries utilisées, nous avons, selon cette méthode, considéré des découpages en 2, 4 et 8 tranches. On trouvera ainsi tableau 2 le calcul des volumes partiels effectués pour le découpage en quatre tranches, et pour un

\begin{tabular}{|c|c|c|c|c|c|c|c|c|c|c|c|c|c|}
\hline \multirow{3}{*}{$\tau$} & \multicolumn{10}{|c|}{$Z=103 \mathrm{~cm}$} & \multicolumn{3}{|c|}{$j z-\underset{\substack{z^{2} \sum \xi_{i} \\
i=1}}{\stackrel{4}{4}}=315,5 \mathrm{~cm}$} \\
\hline & \multicolumn{3}{|c|}{$\begin{array}{c}i=1 \quad h_{1}=16,5 \quad \xi_{1}=1 \\
\tau_{1}=\tau \mathrm{V}_{\infty, 1} / \mathrm{V}_{\infty}=0,2741\end{array}$} & \multicolumn{3}{|c|}{$\begin{array}{ccc}i=2 & h_{2}=20 & \xi_{2}=0,825 \\
\tau_{2}=0,681 \tau \mathrm{V}_{\infty .2} / \mathrm{V}_{\infty}=0,2631\end{array}$} & \multicolumn{3}{|c|}{$\begin{array}{l}i=3 \quad h_{3}=23 \quad \xi_{3}=0,717 \\
\tau_{:}=0,253 \tau \mathrm{V}_{\infty, 3} / \mathrm{V}_{\infty}=0,2535\end{array}$} & \multicolumn{3}{|c|}{$\begin{array}{ll}i=4 & h_{4}=37 \quad \xi_{4}=0,446 \\
\tau_{1}=0,209 \tau \mathrm{V}_{c_{4}} / \mathrm{V}_{\infty}=0,2092\end{array}$} & \multirow{2}{*}{$\mathrm{V}_{w} / \mathrm{V}_{\infty}$} \\
\hline & $\tau_{1}$ & $\left(\mathrm{~V}_{w} / \mathrm{V}_{\infty}\right)_{1}$ & $V_{w, 1} / V_{\infty}$ & $\tau_{2}$ & $\left(V_{w} / V_{s}\right)_{2}$ & $V_{w, 2} / V_{\infty}$ & $\tau_{3}$ & $\left(\mathrm{~V}_{w} / \mathrm{V}_{w}\right)_{3}$ & $V_{w, 3} / V_{\infty}$ & $\tau_{*}$ & $\left(\mathrm{~V}_{w} / \mathrm{V}_{\infty}\right)_{4}$ & $V_{w, d} / V_{\infty}$ & \\
\hline 0,05 & 0,05 & 0,045 & 0,0123 & 0,034 & 0,034 & 0,0093 & 0,026 & 0,025 & 0,0065 & 0,01 & 0,01 & - & 0,028 \\
\hline 0,10 & 0,1 & 0,095 & 0,0260 & 0,068 & 0,068 & 0,0179 & 0,051 & 0,051 & 0,0129 & 0,0199 & 0,020 & 0,005 & 0,062 \\
\hline 0,25 & 0,25 & 0,225 & 0,0617 & 0,17 & 0,16 & 0,0421 & 0,129 & 0,124 & 0,0314 & 0,0497 & 0,050 & 0,0104 & 0,145 \\
\hline 0,50 & 0,5 & 0,39 & 0,107 & 0,34 & 0,29 & 0,0763 & 0,258 & 0,23 & 0,0583 & 0,0995 & 0,098 & 0,0205 & 0,262 \\
\hline 0,75 & 0,75 & 0,53 & 0,145 & 0,51 & 0,40 & 0,1052 & 0,386 & 0,32 & 0,0811 & 0,149 & 0,140 & 0,0293 & 0,360 \\
\hline 1 & 1 & 0,63 & 0,173 & 0,68 & 0,495 & 0,130 & 0,515 & 0,41 & 0,104 & 0,199 & 0,185 & 0,0387 & 0,445 \\
\hline 2 & 2 & 0,865 & 0,237 & 1,36 & 0,745 & 0,196 & 1,03 & 0,65 & $0,16 \overline{5}$ & 0,398 & 0,33 & 0,069 & 0,667 \\
\hline 3 & 3 & 0,95 & 0,260 & 2,04 & 0,87 & 0,229 & 1,54 & 0,79 & 0,200 & 0,696 & 0,455 & 0,095 & 0,784 \\
\hline 5 & 5 & 0,991 & 0,271 & 3,41 & 0,965 & 0,254 & 2,54 & 0,92 & 0,233 & 0,995 & 0,63 & 0,133 & 0,891 \\
\hline
\end{tabular}
rabattement $Z=103 \mathrm{~cm}$ (la valeur de $Z$ ne doit d'ailleurs pas affecter les résultats). Dans ce cas, $\theta_{1}=\theta_{r}$, ce qui fixe un pas de $\Delta \theta=0,067 \mathrm{~cm}^{3} / \mathrm{cm}^{3}$. Les diamètres des familles de tubes sont fixés par:

$$
\begin{gathered}
h_{i}=z_{e}=16,5 \mathrm{~cm} \\
h_{3}=23 \mathrm{~cm}
\end{gathered} \quad h_{.2}=20 \mathrm{~cm}
$$

Tableau 2 / Calcul du volume d'eau drainé dans le cas d'une analogie avec quatre familles de tubes capillaires

Table 2 / Calculated drained water volume for an analogy with four capillary tube families 


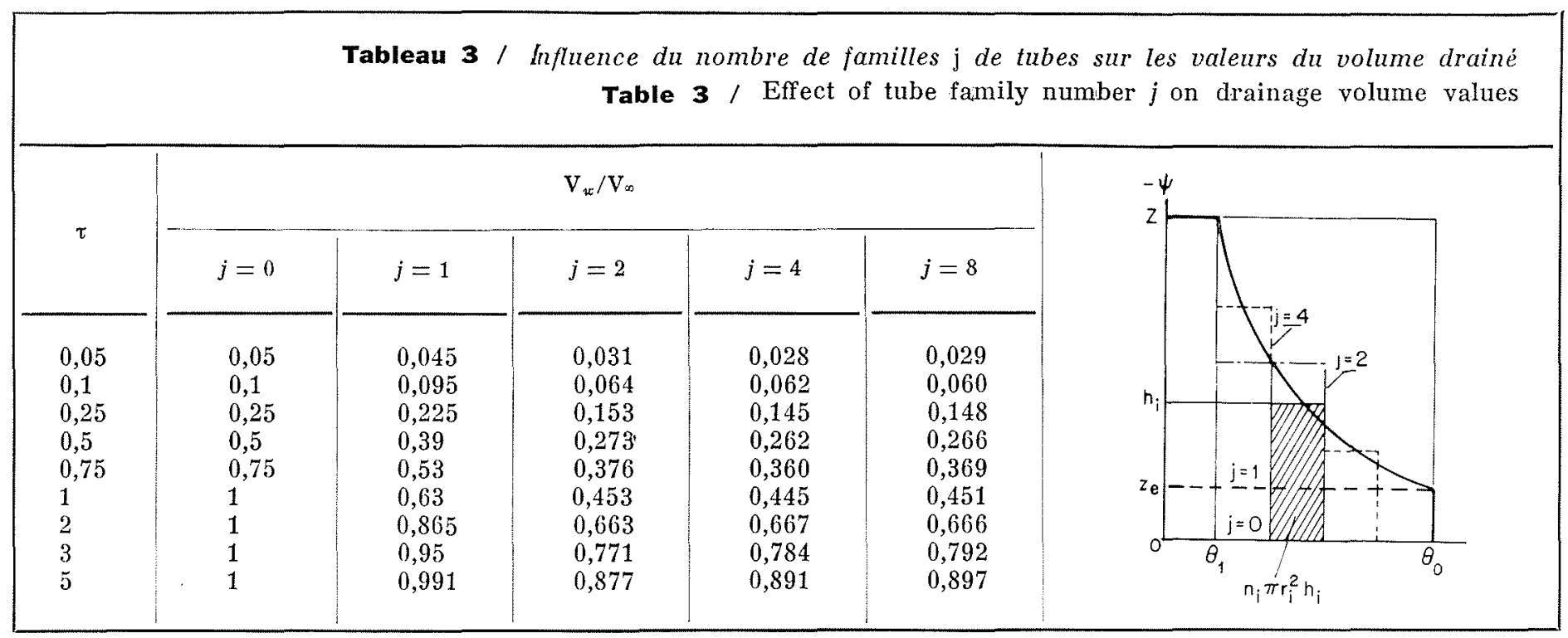

Les résultats obtenus sont également reportés figure 7. On notera que, pour le type de sol considéré, la convergence est extrêmement rapide puisqu'on obtient une courbe unique pour $j=2 ; 4 ; 8$. Notons que le modèle gravitaire correspond à $j=0$. Enfin, dans tous les cas, le temps de référence est bien fixé par analogie avec la famille de tubes de rayon $r_{1}$ correspondant à la hauteur de la frange capillaire $z_{e}$. Les valeurs de $\mathrm{V}_{w} / \mathrm{V}_{\infty}$ pour $j=0,1,2$, 4,8 sont reportées tableau 3 .

La convergence rapide s'explique facilement par l'allure de la courbe d'équilibre du matériau considéré. Passant du cas $j=1$ au cas $j=2$, on passe d'un cas où toute la section d'écoulement est composée de tubes de rayon identique $r_{1}$, à celui où la moitié de la section d'écoulement est composée de tubes de rayon $r_{1}$, l'autre moitié de tubes de rayon $r_{2}=r_{1} / 2$. Soient $n_{1}$ et $r_{1}$ la densité et le rayon des tubes dans le cas 1; on a donc, par surface unitaire de sol :

- pour $j=1$, une section d'écoulement

$$
s=n_{1} \pi r_{1}^{2}
$$

- pour $j=2$, une section d'écoulement

$$
s=\frac{n_{1}}{2} \pi r_{1}^{2}+2 n_{1} \pi \frac{r_{1}^{2}}{4}
$$

Ce qui revient donc à remplacer $n / 2$ tubes de rayon $r_{1}$ par $2 n$ tubes de rayon $r_{1} / 2$.

Le drainage des tubes de rayon $r_{2}=r_{1} / 2$ étant bien plus lent que celui des tubes de rayon $r_{1}$, il en r'ésulte pour le cas $j=2$ des volumes drainés bien plus faibles que dans le cas $j=1$. D'où la variation assez brutale de $V_{u} / V_{\infty}$ passant d'un cas à l'autre. Augmenter ensuite la valeur de $j$ revient à affiner la valeur des volumes obtenus pour $j=2$ sans avoir de grosses variations globales.

Il est enfin intéressant de situer la courbe expérimentale entre les deux modèles. Il est clair que le modèle à tube unique convient bien pour décrire le drainage pour $\tau<0,5$, ce qui, pour $Z=180 \mathrm{~cm}$, correspond à un temps réel de $t=15 \mathrm{mn}$, alors que le modèle à tubes multiples représente assez bien le sol pour $\tau>2$, soit $t=60 \mathrm{mn}$ pour le même rabat- tement. Cette constatation est importante, car elle permet d'établir, s’il est encore utile de préciser ce point, qu'aucun modèle simple de type «capillaire » ne peut représenter l'écoulement en milieux poreux non saturés.

En effet, dans le cas $j=1$, nous avons constaté que l'écoulement s'arrête lorsque le niveau de l'eau dans le tube atteint la cote $z_{\theta}$, ce qui n'est pas le cas dans un sol (fig. 4). Dans le cas $j=2$, le modèle est au contraire relativement satisfaisant lorsque le temps est assez grand, puisque le drainage se poursuit dans les tubes les plus fins après que les tubes les plus gros se soient vidés.

Cependant, le modèle à tubes multiples ne satisfait pas la condition initiale des écoulements en milieux poreux, puisqu'avec un faisceau de capillaires tous les tubes commencent à se vider en même temps alors que, comme le montre la figure $4 a$, les pores les plus fins du sol ne commencent à drainer que lorsque les plus gros, correspondant à une distribution de tubes de rayon $r_{1}$, sont pratiquement déjà vides, ce que confirme la coincidence, pour les faibles temps, entre la courbe expérimentale et la courbe tracée pour $j=1$.

Le seul modèle satisfaisant serait donc en fait celui où la distribution des familles de tubes $n_{i}, r_{i}$ varierait avec le temps, passant d'une distribution $j=1$ de tubes uniformes, de section d'écoulement $n_{1} \pi r_{1}{ }^{2}$ en début de drainage, à une distribution multiple $j_{n}$ au fur et à mesure de l'évolution du drainage, la loi $j(t)$ étant une caractéristique du matériau, fonction du rabattement et du temps, donc analogue au coefficient d'emmagasinement.

Signalons cependant que les deux courbes $j=1$, $\mathrm{j}=n$, peuvent être utilisées comme les limites de la courbe expérimentale.

Après cette diversion sur les modèles, il est intéressant d'étudier, pour un type de sol considéré, la variation du coefficient d'emmagasinement en fonction du temps, du rabattement et de la profondeur de la nappe. Le tableau 1 permet d'obtenir facilement, à partir des mesures de volume d'eau écoulé, la valeur de ce coefficient pour les essais de drainage étudiés. La loi empirique $\mathrm{S}=f(Z, t)$ pour $H=0$ est reportée figure 8 . Notons simplement 
qu'au début du drainage, pour $t<20 \mathrm{mn}$, S diminue lorsque $Z$ augmente, alors qu'à partir d'un temps critique qui vaul approximativement $30 \mathrm{mn}$, $S$ est, à un instant donné, pratiquement indépendant du rabatlement sitôt que celui-ci est suffisamment important.

La courbe limite, correspondant à $\mathrm{V}_{w}=\mathrm{V}_{\infty}$, est l'enveloppe du réseau, et tend asymptotiquement vers $\varepsilon$. La forme de la courbe d'équilibre ne dépend que de la courbe de succion, alors que la vitesse avec laquelle $S$ tend rers $\varepsilon$ dépend essentiellement de la loi $k(\theta)$.

Comme nous l'avons exposé précédemment (Vachaud, $1967 a)$, il est enfin normal que, pour la mème valeur du rabattement, la valeur finale de $\mathrm{S}$ soil d'autant plus proche de $\varepsilon$ que la profondeur de la nappe est importante. A partir du moment où $\mathrm{H}$ est tel que les courbes d'équilibre 1 et 2 de la figure $1 \mathrm{~b}$ se déduisent totalement par translation $Z$, on doil même avoir $S_{1 i n}=\varepsilon$. Pour le sol considéré, celte profondeur critique (fig. 2) vaut : $\mathrm{H}_{c} \sim 65 \mathrm{~cm}$. Ceci apparait si l'on calcule d'après le tableau 1 , les valeurs de $S$ obtenues pour $H=0, Z=103 \mathrm{~cm} \mathrm{et}$ $\mathrm{H}=103 \mathrm{~cm}, Z=77 \mathrm{~cm}$. Dans ce dernier cas, on obtient bien $S_{l i n}=\varepsilon$.

Enfin, le drainage étant contròlé par la conductivité capillaire à la surface du sol, et par le volume d'eau à drainer, il est naturel que l'écoulement soit d'autant plus long que le rabattement est important.

On peut, pour chaque rabattement, définir la durée du drainage comme le temps nécessaire pour que $99 \%$ du volume total se soit écoulé; soit $t_{99}$ ce temps. La courbe en pointillés figure 5 est obtenue en reliant pour les différents essais les points représentant $\mathrm{V}_{w} / \mathrm{V}_{\infty}=0,99$. La loi $t_{99}=f(Z)$ est reporlée figure 9. Cette loi a une allure exponentielle lorsque le rabattement est suffisant, mais, comme aucun drainage n'intervient pour $Z \leqslant z_{\theta}$, on doil avoir $t_{99} \rightarrow 0$ pour $Z \rightarrow z_{e}$, pour un drainage de surface.

La pente de la droite représentant la relation $t_{99}=f(Z)$ en coordonnées semi-logarithmiques doit être une fonction de $K_{0}$. Cette courbe a été entourée par les résultats obtenus par Prill et Johnson (1965) pour les essais de drainage effectués sur les sols suivants :

- Del Monte Sand :

$\mathrm{K}_{0}=9,3 \mathrm{~cm} / \mathrm{mn} \quad Z=151 \mathrm{~cm} \quad z_{c}=10 \mathrm{~cm}$;

_. $0,120 \mathrm{~mm}$ Glass Beads :

$\mathrm{K}_{0}=0,86 \mathrm{~cm} / \mathrm{mn} \quad Z=171 \mathrm{~cm} \quad z_{c}=53 \mathrm{~cm}$;

-_- Fresno Medium Sand :

$\mathrm{K}_{0}=0,46 \mathrm{~cm} / \mathrm{mn} \quad Z=151 \mathrm{~cm} \quad z_{e}=12 \mathrm{~cm}$.

On trouvera également figure 9 la loi $t_{99}=f(Z)$ obtenue pour un gravier grossier $(d>2 \mathrm{~mm})$, qui est le complément du sable de rivière utilisé pour ce mémoire. Ce gravier est caractérisé par :

$$
\mathrm{K}_{0}=9 \mathrm{~cm} / \mathrm{mn} \quad z_{\mathrm{c}}=2,5 \mathrm{~cm}
$$

Les résultats des essais de drainage effectués selon la mème méthode sur ce gravier, sont reportés dans Vachaud $(1967 \mathrm{c})$.

La simple mesure des volumes d'eau écoulés pendant le drainage de colonne de sol permet donc déjà de contrôler la valeur des hypothèses couramment adoptées, de juger de la validité des modèles à représenter ce type d'écoulement et d'estimer les

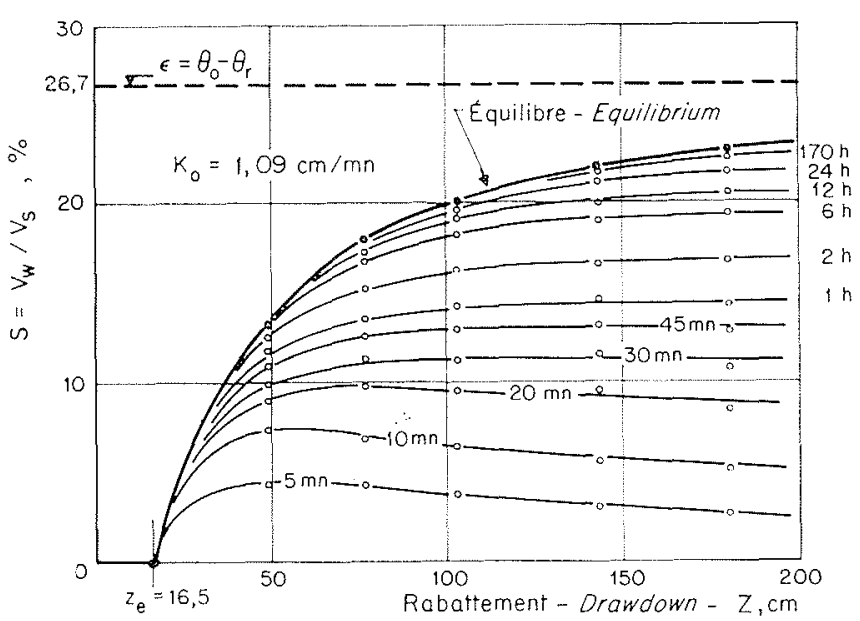

$8 /$ Variation du coefficient d'emmagasinement en fonction du rabattement et du temps pour les drainages de surface.

Variation of storatge coefficient with drawdown and time for surface drainage.

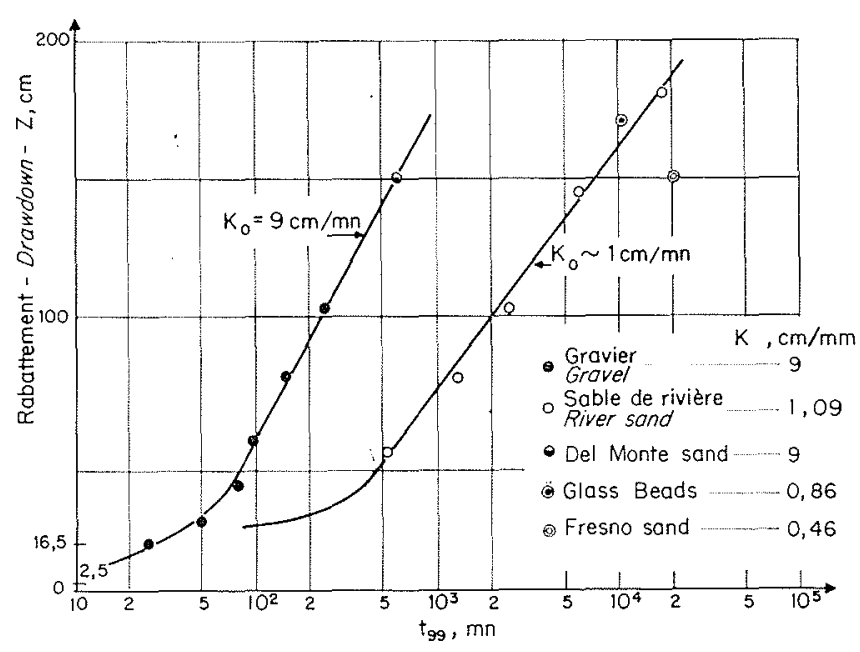

9/ Estimation de la durée du drainage en fonction du rabaltement et de la perméabilité du sol.

Estimated drainage duration vs, drawdown and soil permeability.

variations du coefficient d'emmagasinement et la durée du drainage. Il est cependant nécessaire de faire un pas supplémentaire pour étudier complètement le problème du drainage : analyser l'évolution des teneurs en eau et étudier la physique de l'écoulement dans la zone non saturée.

\section{IV. - Analyse des profils hydriques Détermination de la conductivité capillaire}

La résolution numérique de l'équation (7) nécessite la connaissance de la loi $k(\theta)$, et la vérification de l'hypothèse de linéarité entre les débits et les gradients de potentiel.

En utilisant le mème procédé que celui développé précédemment (Vachaud, $1966 \mathrm{~b}$ et $1967 \mathrm{~b}$ ), l'analyse de l'évolution des teneurs en eau durant le 


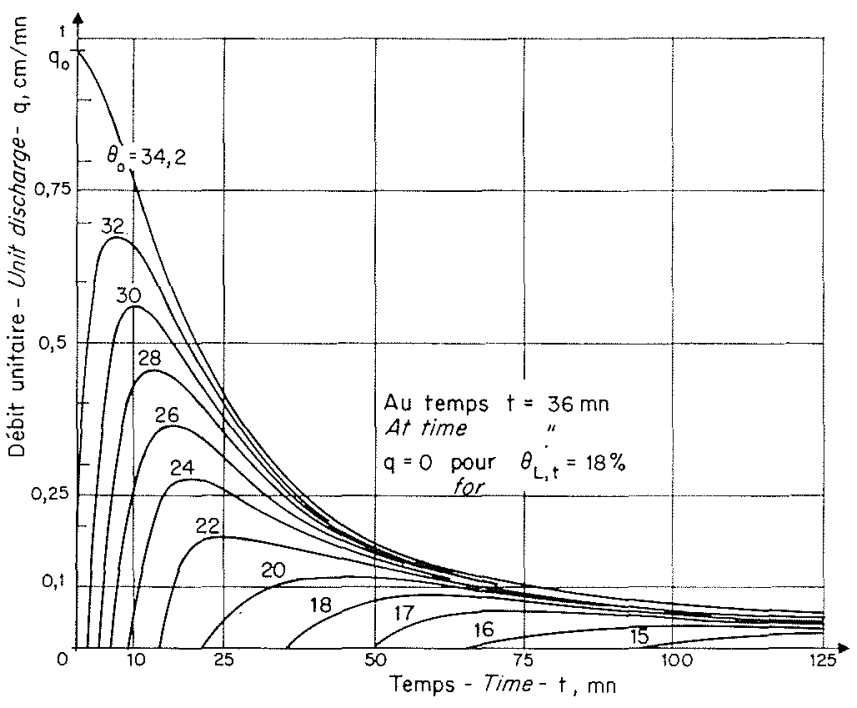

10/ Drainage $H=0, Z=180 \mathrm{~cm}$. Variation du flux $q$ en fonction du temps. Paramètre $=$ teneur en eau $\theta$, \% Drainage $H=0, Z=180 \mathrm{~cm}$. Variation of flux q with time. Parameter: water content $\theta, \%$.

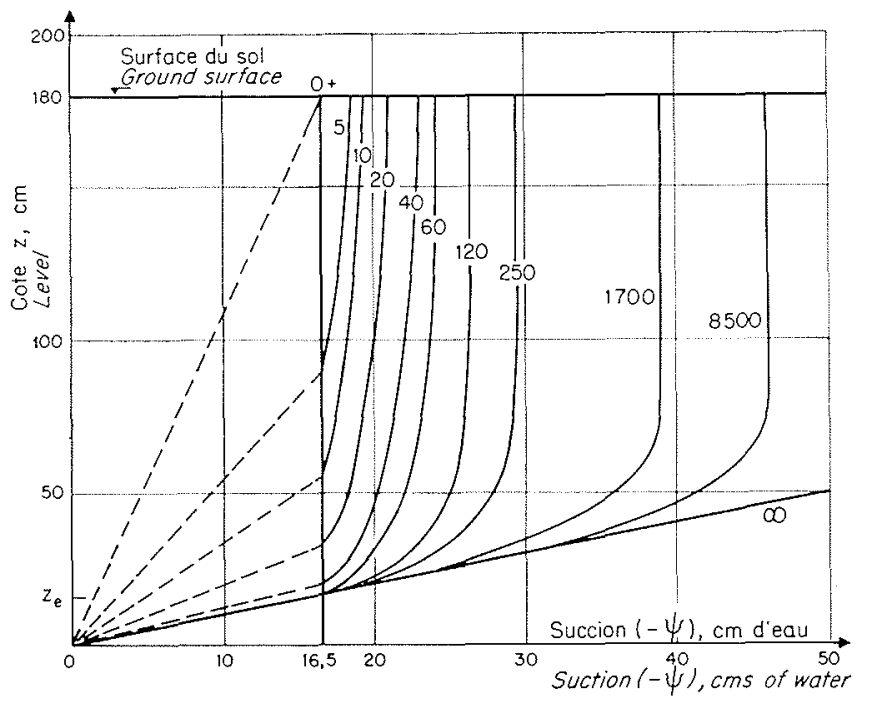

11/ Drainage $\mathrm{H}=0, \mathrm{Z}=180 \mathrm{~cm}$. Distribution de la succion durant le drainage. Paramètre $=$ temps $t$ en minutes. Drainage $H=0, \quad Z=180 \mathrm{~cm}$. Suction distribution during drainage. Parameter: time $t$ in minutes. drainage permet d'établir la relation $k(\theta)$ et de montrer que cette relation est unique, pour le matériau et le type d'écoulement considéré.

La loi de Darcy généralisée s'écrit:

$$
q=-k(\theta)\left(\frac{\partial \psi}{\partial z}+1\right)
$$

Si cette loi est valable, la valeur de $k$ doit être unique, pour une teneur en eau déterminée $\theta_{n}$ quels que soient les gradients de potentiel et les débits unitaires.

Le débit unitaire $q$ doit en outre satisfaire l'équation de continuité :

$$
\operatorname{div} q=-\frac{\partial \theta}{\partial t}
$$

chaque tranche de sol d'épaisseur $\Delta z$ se comportant, duranl l'écoulement, comme une zone de transmission avec perte de masse: la différence entre le débit entrant et le débit sortant de cette tranche provenant d'une diminution du volume d'eau stocké dans cetle tranche.

Si l'on considère qu'entre les instants $t$ et $t+d l$ la variation de teneur en eau de la tranche $[z$, $z+d z]$ est faible, et si l'on appelle $\theta_{n}$ la valeur moyenne de $\theta$ durant cet intervalle, avec $d \theta_{n}=0$, (14) s'écrit, pour un écoulement unidimensionnel :

$$
\frac{\partial q}{\partial z}=\frac{\partial \theta}{\partial z} \cdot \frac{d z}{d t}
$$

Soit $v=(d z / d t)$ la vitesse d'avancement de la teneur en eau; si entre deux valeurs voisines $\theta_{k+1}$ et $\theta_{k}$ de la teneur en eau $v$ varie peu - soit $\bar{v}_{k}$ sa valeur moyenne - on peut intégrer (15) sous la forme :

$$
q_{k+1}-q_{i k}=\bar{v}_{k}\left(\theta_{k+1}-\theta_{k}\right)
$$

Le calcul des débits s'effectue donc par un procédé itératif.

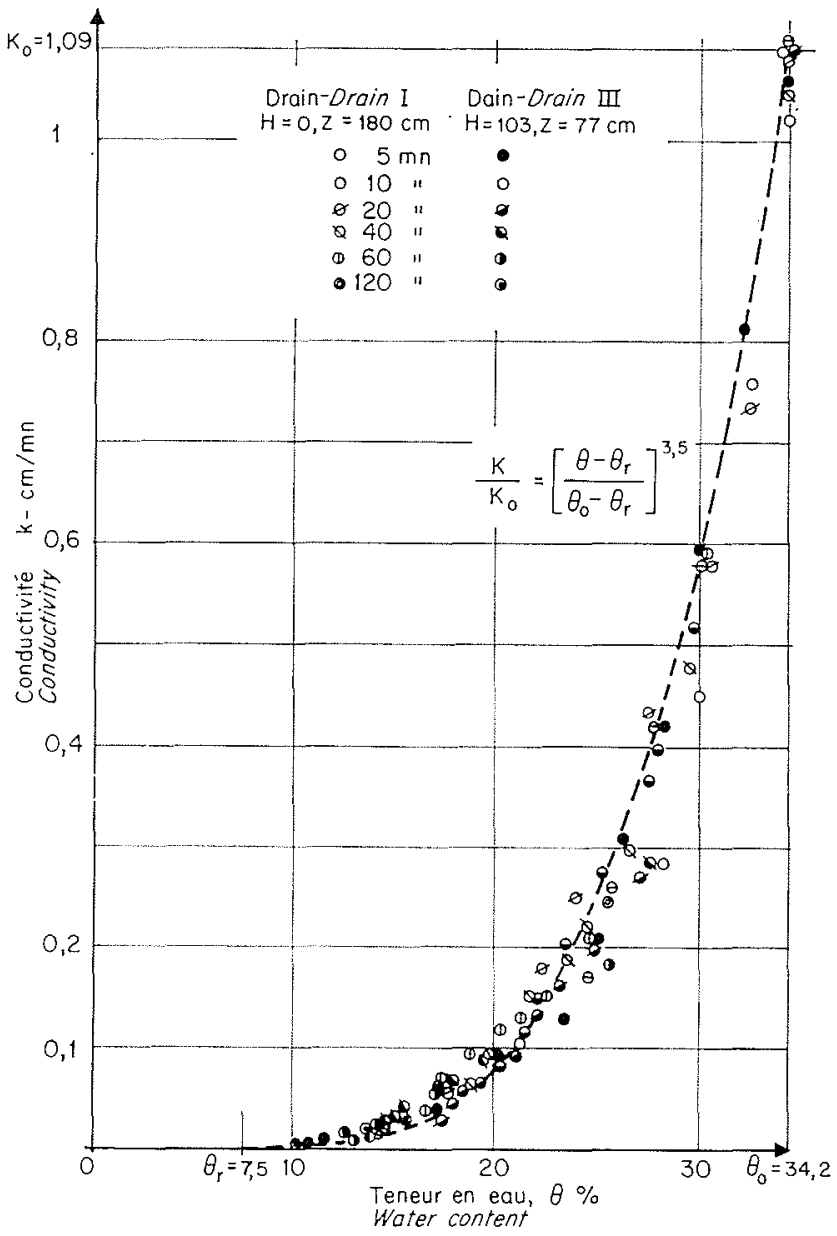

12/ Courbe $k(\theta)$. La courbe en pointillé correspond à la formule (17).

$\mathrm{k}(\theta)$ curve. The alashed curve refers to equation (17). 
Le gradient de potentiel $\partial / \partial z(\psi+z)$ est obtenu en utilisant la courbe de pression capillaire $\psi(\theta)$ déterminée à part sur un échantillon de même sol, et obtenue également à l'équilibre pour le drainage III. La mesure des profils hydriques donne l'évolution de la teneur en eau $\theta$ à une cote $z_{n}$ en fonction du temps. En utilisant la courbe $\psi(\theta)$ on peut donc facilement obtenir l'évolution de $\left(\psi+z_{n}\right)$ à la cote $z_{n}$ en fonction du temps.

En groupant les couples de valeurs $q$ et $\partial / \partial z(\psi+z)$ on peut alors calculer $k$.

Cette méthode d'analyse a déjà été longuement exposée dans des publications précédentes (Vachaud, 1966 et 1967 b), nous ne reviendrons pas sur le calcul détaillé des différentes fonctions. On trouvera figure 10 l'évolution des débits unitaires et figure 11 celle des gradients de potentiels durant le drainage I. Le gradient de potentiel est constant sous le niveau du front et varie, à la cote $z=\mathrm{L}$, de $0,909(t=0)$ à 1 . Les courbes en pointillés figure 11 donnent l'évolution du gradient durant la période de drainage lent, une fois le front stabilisé à la cote $z=z_{c}(t>60 \mathrm{mn})$.
Les couples de valeurs $q$ et $\partial \Phi / \partial z$ sont regroupes tableau 4 pour différentes valeurs de $t$ et $z$. La courbe $k(\theta)$ obtenue en regroupant des couples est reportée figure 12 . On trouvera également figure 12 les valeurs de $k(\theta)$ obtenues par analyse du drainage III.

Il est clair que $k(\theta)$ est une loi unique pour ce sol, quelles que soient les valeurs de $q$ et $\partial \Phi / \partial z$, et indépendamment des conditions aux limites de l'écoulement. L'hypothèse de généralisation de la loi de Darcy peut donc être considérée comme valide.

La courbe tracée en pointillés figure 12 est une courbe empirique de variation de $k(\theta)$, obtenue par :

$$
k(\theta)=\mathbf{K}_{0}\left(\frac{\theta-\theta_{r}}{\theta_{0}-\theta_{r}}\right)^{3, \pi}
$$

selon la méthode proposée par Boreli-Vachaud, 1966.

Cette courbe représente relativement bien la loi $k(\theta)$, sauf dans les zones de faible teneur en eau $(\theta<15 \%)$.

\begin{tabular}{|c|c|c|c|c|c|c|c|c|c|c|c|c|}
\hline & & & & & \multicolumn{8}{|c|}{$\begin{array}{l}\text { Tableau } 4 / \text { Drainage } \mathrm{H}=0, \mathrm{Z}=180 \mathrm{~cm} \text {. Calcul de } k(\theta) \\
\text { Table } 4 / \text { Drainage } \mathrm{H}=0, \mathrm{Z}=180 \mathrm{~cm} \text {. Calculated } k(\theta)\end{array}$} \\
\hline & \multicolumn{4}{|c|}{$5 \mathrm{mn}$} & \multicolumn{4}{|c|}{$10 \mathrm{mn}$} & \multicolumn{4}{|c|}{$20 \mathrm{mn}$} \\
\hline $\begin{array}{l}z \\
(\mathrm{~cm}) \\
\end{array}$ & $(\stackrel{\theta}{\%})$ & $\frac{q}{(\mathrm{~cm} / \mathrm{mn})}$ & $d \Phi / d z$ & $\begin{array}{c}k \\
(\mathrm{~cm} / \mathrm{mn})\end{array}$ & $\stackrel{\theta}{\%}$ & $\begin{array}{c}q \\
(\mathrm{~cm} / \mathrm{mn})\end{array}$ & $d \Phi / d z$ & $\begin{array}{c}k \\
(\mathrm{~cm} / \mathrm{mn})\end{array}$ & $\left(\begin{array}{c}\theta \\
\%\end{array}\right)$ & $\frac{q}{(\mathrm{~cm} / \mathrm{mn})}$ & $d \Phi / d z$ & $\begin{array}{c}k \\
(\mathrm{~cm} / \mathrm{mn})\end{array}$ \\
\hline $\begin{array}{r}180 \\
150 \\
125 \\
100 \\
75 \\
50 \\
40 \\
35 \\
30 \\
25 \\
20\end{array}$ & $\begin{array}{l}27 \\
28,2 \\
30 \\
32,5 \\
34,2\end{array}$ & $\begin{array}{l}0,28 \\
0,44 \\
0,72 \\
0,895\end{array}$ & $\begin{array}{l}0,99 \\
0,98 \\
0,974 \\
0,95 \\
0,82\end{array}$ & $\begin{array}{l}0,29 \\
0,45 \\
0,758 \\
1,092\end{array}$ & $\begin{array}{l}23,5 \\
24,6 \\
25,8 \\
27,8 \\
30 \\
34,2\end{array}$ & $\begin{array}{l}0,17 \\
0,255 \\
0,41 \\
0,56 \\
0,77\end{array}$ & $\begin{array}{l}0,99 \\
0,988 \\
0,986 \\
0,98 \\
0,965 \\
0,70\end{array}$ & $\begin{array}{l}0,172 \\
0,26 \\
0,42 \\
0,58 \\
1,10\end{array}$ & $\begin{array}{l}20,3 \\
20,6 \\
21,2 \\
22,2 \\
24 \\
27,6 \\
30,4 \\
32,4 \\
34,2\end{array}$ & $\begin{array}{l}0,07 \\
0,102 \\
0,176 \\
0,24 \\
0,396 \\
0,452 \\
0,484 \\
0,51\end{array}$ & $\begin{array}{l}0,995 \\
0,99 \\
0,988 \\
0,98 \\
0,97 \\
0,91 \\
0,78 \\
0,66 \\
0,47\end{array}$ & $\begin{array}{l}0,071 \\
0,103 \\
0,18 \\
0,25 \\
0,436 \\
0,58 \\
0,734 \\
1,082\end{array}$ \\
\hline & \multicolumn{4}{|c|}{$40 \mathrm{mn}$} & \multicolumn{4}{|c|}{$60 \mathrm{mn}$} & \multicolumn{4}{|c|}{$120 \mathrm{mn}$} \\
\hline $\begin{array}{l}z \\
(\mathrm{~cm})\end{array}$ & $\stackrel{\theta}{(\%)}$ & $\begin{array}{c}q \\
(\mathrm{~cm} / \mathrm{mn})\end{array}$ & $d_{\varphi} / d z$ & $\begin{array}{c}k \\
(\mathrm{~cm} / \mathrm{mn})\end{array}$ & $\stackrel{\theta}{(\%)}$ & $\underset{(\mathrm{cm} / \mathrm{mn})}{q}$ & $d_{\varphi} / d z$ & $\begin{array}{c}k \\
(\mathrm{~cm} / \mathrm{mn})\end{array}$ & $\begin{array}{c}\theta \\
(\%)\end{array}$ & $\begin{array}{c}q \\
(\mathrm{~cm} / \mathrm{mn})\end{array}$ & $d_{\varphi} / d z$ & $\begin{array}{c}k \\
(\mathrm{~cm} / \mathrm{mn})\end{array}$ \\
\hline $\begin{array}{l}180 \\
150 \\
125\end{array}$ & 17,6 & 0,054 & 1 & 0,054 & 16,5 & 0,04 & 1 & 0,04 & 14,6 & 0,021 & 1 & 0,021 \\
\hline 100 & 18,7 & 0,064 & 0,985 & 0,065 & 16,7 & 0,04 & 0,995 & 0,04 & & & & \\
\hline 75 & 19,6 & 0,098 & 0,975 & 0,099 & 17,2 & 0,07 & 0,98 & 0,071 & 14,8 & 0,022 & 0,965 & 0,023 \\
\hline 50 & 21,5 & 0,144 & 0,945 & 0,152 & 18,8 & 0,096 & 0,96 & 0,100 & 15,5 & 0,027 & 0,895 & 0,030 \\
\hline 40 & 23,4 & 0,168 & 0,88 & 0,191 & 20,2 & 0,106 & 0,905 & 0,117 & 16,4 & 0,036 & 0,845 & 0,043 \\
\hline 35 & 24,4 & 0,182 & 0,81 & 0,225 & 21,2 & 0,112 & 0,88 & 0,130 & 17,4 & 0,042 & 0,80 & 0,052 \\
\hline 30 & 26,4 & 0,198 & 0,66 & 0,300 & 22,5 & 0,118 & 0,77 & 0,153 & 18,7 & 0,046 & 0,70 & 0,065 \\
\hline 25 & 29,4 & 0,216 & 0,45 & 0,48 & 24,6 & 0,123 & 0,59 & 0,208 & 21 & 0,051 & 0,52 & 0,098 \\
\hline 20 & 34,2 & 0,235 & 0,225 & 1,045 & 30,2 & 0,133 & 0,225 & 0,592 & 25,4 & 0,056 & 0,23 & 0,244 \\
\hline
\end{tabular}




\section{$V_{*}$ - Conclusion}

Nous avonc donc exposé une méthode expérimentale qui permet d'analyser la validité de deux hypothèses de base couramment utilisées dans les problèmes d'écoulement en milieux poreux non saturés. La première concerne la défnition du coefficient d'emmagasinement; la seconde est relative à la théorie générale des éconlements puisqu'elle revient à exprimer une relation de linéarité entre les flux et les potentiels.

Les résultats concernant le premier point montrent clairement que l'identité $S \equiv \varepsilon$, associée avec le modèle gravitaire [cf. équation $\left.\left(1^{\prime}\right)\right]$, conduit assurément à des estimations bien trop fortes des volumes d'eau drainés.

Les modèles de type capillaire, formés par une série uniforme de tubes de rayon $r_{1}$, associés à la hauteur de la frange capillaire, ou des séries multiples de $n_{i}$ tubes de rayon $r_{i}$, conduisent à des estimations plus favorables. Mais il faut noter que le drainage d'une nappe n'est pas un phénomène aussi simple que le drainage d'un tube capillaire, ou d'une série de tubes. Dans ce dernier cas en effet, tous les tubes commencent à drainer en même temps, alors que l'on peut grossièrement caractériser le drainage d'une nappe par deux phases successives :

- dans une première période - au début de l'écoulement —. le sol peut ètre associé à une série de tubes uniformes de rayon $r_{1}$. Le débit est alors contròlé par la position du front de teneur en eau $\theta_{0}$, et l'analogie avec le modèle $j=1$ est satisfaisante. Pour le sol considéré, la durée de cette période vaut, en temps réduit, $\tau \sim 0,5$. Selon la granulométrie et la perméabilité du sol, cette durée pourra être plus ou moins étendue;

- dans une seconde période, qui commence lorsque le front se rapproche de sa valeur d'équilibre, on doit considérer l'analogie avec un modèle formé de plusieurs familles de tubes de rayon $r_{i}<r_{1}$. Le volume drainé est équivalent à celui domné par ce modèle à tubes multiples pour $\tau>2$, pour le sol considéré.

Pour $0,5<\tau<2$, il faudrait pouvoir imaginer un modèle où la densité de tube varie graduellement d'un modèle formé uniquement par des tubes de rayon $r_{1}$, pour $\tau<0,5$, à un modèle formé uniquement de tubes très fins.

Le drainage dure d'autant plus que le rabattement est long, et le sol fin. On notera, d'après les courbes données figure 9 , que pour le sol considéré, il faut attendre plus de 15 jours pour que $99 \%$ du volume drainable se soit écoulé pour un rabattement de $180 \mathrm{~cm}$. Si l'on se reporte à la figure $4 a$, on voit notamment que, pour ce rabattement, durant la seconde période de drainage considérée ci-avant (pour $\tau>2$, soit $t>60 \mathrm{mn}$ ), les gradients de potentiel deviennent négligeables à la base de la colonne, dans la zone où les profils hydriques se rapprochent de la courbe d'écuilibre, alors que ces gradients valent 1 à la surface du sol. Au fur et à mesure que l'écoulement progresse, c'est donc la valeur de la conductivité capillaire $k$, correspondant à la teneur en eau à la surface du sol, qui tend à fixer la valeur du débit drainé, les tranches inférieures de la colonne se bornant à transmettre les volumes d'eau recus sans apport complémentaire.

L'analyse des profils hydriques oblenus par absorption de rayons $\gamma$ permel enfin de calculer la conductivité hydraulique el de vérifier la validite de la loi de Darcy généraliscé, sauf peul-être au début de l'écoulement qui reste inacessible à la mesure.

Connaissant la loi $k(0)$, il devient alors possible d'obtenir, par résolution de l'équation (7), l'érolulion de $\theta(z, t)$ et de $\Phi(z, t)$ sous forme de données numériques pour un essai de drainage quelconque. Nous sommes actuellement en train de mettre au point un procédé numérique de résolution de cette équation (Guélin, 1967).

$$
\because
$$

Cet article recouve en parlie le travail d'une thèse de Doctorat d'Etat es Sciences Physiques à soutenir devant la Faculté des Sciences de Grenoble.

Les essais ont été effectués aux Laboratoires de Mécanique des Fluides de l'Université de Grenoble. L'auteur est particulièrement reconnaissant à M. le Professeur L. Santon d'avoir bien voulu accepter de diriger ce travail, et il lui sail gré d'avoir toujours été disposé à lui apporter conseils et aide matérielle. Il tient également à exprimer sa gralitude à M. B. Wack et M. P. Guélin pour leur amicale participation à de nombreuses discussions concernant celte recherche.

Manuseril recu le 28 fébrier 1968

\section{Liste dies symboles}

A : section de la colonne......... I

D : coefficient de diffusitivité...... L $\mathrm{L}^{2} \cdot \mathrm{T}^{-1}$

$d$ : diamètre des grains........... L

$\mathrm{H}$ : distance entre la nappe et la surface du sol avant le début diu drainage.............. I

$h_{i}$ : remontée capillaire dans un tube de rayon $r_{i} \ldots \ldots \ldots \ldots \ldots$ I

$K_{0}$ : perméabilité saturée du sol...... L. T-1

$k$ : conductivité hydraulique, fonction

de la teneur en eau.......... L. . T

$L$ : cote du sommet de la colonne... L L

$\mathrm{N}_{0}$ : taux de complage de référence... . $\mathrm{T}^{-1}$

$N$ : taux de complase apres passage des rayons $\gamma$ dans le sol........ $\mathrm{T}^{-1}$

$n$ : porosité.............. L L $\mathrm{L}^{3} \mathrm{~L}^{3}$ ou \%

$q_{10}$ : flux à l'instant initial du drainage. L.T-1

q: Mux, fonction de la teneur en eau et du temps............. I. T-1.

$S$ : coefficient d'emmagasinement... \%

$t:$ temps.............. T

$V_{11}:$ volume d'eau drainé. . . . . . . L

$V_{s}:$ volume de sol drainé. . . . . . L $\mathrm{L}^{3}$

$\mathrm{V}_{\infty}$ : volume d'eau drainé à l'équilibre. $\mathrm{L}^{3}$

$v$ : vilesse d'avancement des profils.. L. T-1

wo : teneur en eau pondérale........ M.M-1

$x$ : épaisseur de la colonne de sol... I I

$z$ : cote au-dessus du fond de la colonne............... I

$z_{e}$ : épaisseur de la frange capillaire... L 
$Z$ : rabaltement. . ..........

$\varepsilon$ : porosité effective. ...........

$\eta$ : viscosité dynamique. . . . . . . .

$\mu_{w}$ : coefficient d'absorption massique de l'catl. . . . . . . . . . . . . .

$\mu_{s}$ : coefficient d'absorption massique du sol see. . . ...........

$\theta$ : teneur en eat rolumique du sol.

$\theta_{0}$ : teneur en eau sous le niveau de la nappe. . . ...........

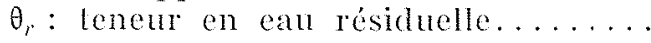

$\Phi$ : polentiel total $(\downarrow+z) \ldots \ldots \ldots \ldots$

$\Psi$ : succion $(\psi$ est négatip) .........

$\rho_{n}$ : masse volumique de l'eau........

$P_{l}$ : masse rolumique du sol sec.....

$\tau:$ temps réduit $=\left(A\left(\gamma_{0} / V_{\infty}\right) . t \ldots \ldots\right.$

$\xi$ : hauteur réduite $=\left(h_{i} / z_{0}\right) \ldots \ldots \ldots$
L

$\%$

N. $\mathrm{L}^{-1} \cdot \mathrm{T}^{-1}$

$L^{\prime} \cdot M^{-1}$

$\mathrm{L}^{2} \cdot \mathrm{MI}^{-1}$

$\mathrm{I}: \mathrm{L} \cdot \mathrm{L}-3$ on $\%$

$\mathrm{I}^{3} \cdot \mathrm{I}-\mathrm{s}$

I

I.

M.I-3

M.L-:

adim.

$\gg$

\section{Pibliographie.}

Benblida (a.). - Etude expérimentale de quelques problèmes d'ecotement en miliewx poreux non saturés par des procédes de mesure radioactifs. Thèse de $3^{\circ}$ Cycle, Laboratoire de Mecanique des Fluides, Grenoble (1963).

Boner (M.) et Vachacb (G.). - Note sur la détermination de la teneur en ean résiduelle et sur la varation de la perméabilité relative dans les sols non saturés. C.R. Ac. Soi. Paris, t. $263,(1966), 698-701$.

Boywer (J.) et rav Schlmeiande (J.). - Simplified method of predicting the fall of water table in drained land. Trans. ASAE, rol. 6, (1963), 228-291.

Cmuns (E. C.). - The non steady state of the water table in drained land. J. of Geophys. Res., vol. 65, (1960), $780-782$.

Chros (E. C.) et Pourovassmas (A.). - The moisture profiles above a moving water table. J. of Soil Sci., rol, 13 , $\mathrm{n}^{\circ 2} 2,(1962), 271-285$.

Croney (D), Comman (I. D.) et Brack (W. P.) - Movement and Distribution of Water in Soil in retation to Highway Design and Performance. Highumal Res. Board, Spec. Rep. 40, (1958), 226-252.

DAvidsox (J. M.), Bigiar (I. W.), et NiELSeN (D. R.). Gamma-radiation attenuation for measuring bulk densify and transient flow in porous media. I. of Geophys Res., vol. $68,(1963),+777-4783$.

DAY (P.) et LeTHIN (J.N.). - A numerical solution of the differential equation of flow for a vertical drainage problem. Proe. Soil Sci. Soe. of Am., vol. 20, (1956), 443447 .

Degatenen (R.). - Mecanisme de lanimentation des nappes souteraines. Chronique d"Hydrogéologie, n" 8 (1960), $85-98$.

Fenris (G.) et Koowress (D.B.). - - Theory of Aquifer Test U.S.G.S., Water Supply Paper 1536-E. (1962).

Fusoka (Y.) et KrTanuma (T.) - Approximate solution of a vertieal drainage problem. J. of Geophys. Res., vol. $69,(1964), 5249-5255$.

Gardener (W. R.). - Approximnte solution of a non-steady drainage problem. Proce Soil Sci. Soce of Am., vol. 26 (1962), 129-132.

Guerrx (P.). - Intégration d'une équation de diffusion comme préliminaire à la situation numérique des écoulements de fluide en milien poreux non saturé C.R. Ac. Sci. Paris, rol. 265, (1967), 876-879,

Havese (A.S.). - Analysis of data from pumping tests in anisotmonic aquifers. $r$. of Geophys. Res., rol. 71 , $n^{\circ} 2,(1966), 421-426$.
JExsex (M. E.) et Haxis (R. J.). -- Non steedy state drainage from porous media. Proce A.S.C.E., J. of the Irrigation and Drainage Div., vol. 93, IIR 3, (1967), 209-231.

Lakopoulos (A.C.). - Theoretical solution of the unsteady unsaturated flow problems in soils. Bulletin de l'A.I.H.S., vol. $10, \mathrm{n}^{\prime \prime} 1,(1965), 5-39$.

LeTHIX (J.N.) et Wonsteld (R.V.). - The falling water table in tile drainage. A laboratory study. Proc. Soil Sci. Soc of Am., rol. 21, (1957), 580-584.

Menzer (C.E.). - Outline of methods for estimating ground water supplies. U.S.G.S., Waler Supply Paper, 638-C, (1932).

Niblosen (D. R.) el Yachaud (G.). - Infiltration of water into vertical and horizontal soils columns. $J$. of Indian Soc. of Soil science, vol. 13, (1964), 15-23.

PHirfi (J.R.). - The theory of infiltration, 1, soil science, vol. $83,(1958), 345-357$.

Prill (R. C.), Johnson (A. I.) et Momms (D. A.). - - Specific yield-laboratory experiments showing the effect of time on column dminage. U.S.G.S., Water Supply Paper $1662-13(1965)$.

Reginato (R.J.) et van Bavel (C. H. M). - Soil Water Measurement with Gamma Attenuation. Proc. Soil Sci. Soce of $A m$, vol. 28 (1964), 721-724.

Renson (I.) et Drake (R.L.) et al. -- Vertical drainage of an unsaturated soil. Proc. ASCE, J. of the IIydranlics Division, vol. 91, HY-1, (1965), 55-74.

Remson (I.) et al. - Numerieal Analysis of Soil Moisture Systems, Proc. ASCE, vol. 93, IR-3, (1967), 153-166.

Van Schilfagande (J.). - Theory of Land drainage, in Drainage of Agricultural Land, J.N. Luthis, (1957), $97-107$.

TAron (G.S.), - Drainable porosity evaluation from outflow measurement and its use in drawdown equation. Soil Sci., vol. 90, (1960), 338-343.

Tond (D. K.). - Gromel Water Hydrology. J. Wiley and Sons, Ed. $(1957)$.

Topr (G. C.) et Mrenen (E. E.). - Hysteretic Moisture Characteristics and hydraulic conductivities for glass bend media. Soil Sci. Soce of $4 \mathrm{~m}$. Proce, vol. 30, (1966), 156162.

Vachatd (G.). - Vérifieation de la loi de Darey généralisée et determination de la conductirité capillaire à partir d'une infiltration horizontale. Intern. Symposium on water in the Unsalurated Zone, Wageningen, June 19-25, (1966), Ib-2.

Vachavo (G.). - a a). Etude de la valeur du coefficient d'emmagasinement des nappes ì surface libre. Symp. Intern. de l'Aménagement des nappes (Haîfa). Public. $n^{\circ} 72$ de l'A.I.H.S., (1967), 69-82.

Vachaun $\left.\left(x_{x}\right),-b\right)$. Determination of the hydraulic conductivity of unsalurated soils from an analysis of transient flow data. Water Resources Res., vol. 3, (1967), 697-705.

Vachayn (G.). - c). Transfert de l'eau dans les sols non saturés. 86" Congrès AFAS Bordeaux (en cours de publi(ation) (1967).

Wack (B.). - Nesure de la densité et de l'humidité des sols par radiontivité. Rapport interne, Laboratoire de Nécanique des Fluides, Grenoble (1961).

Watson (K. K.). - An instantaneous profile method for determining the hydraulic conductivity of unsaturated porous materials. Water Resources Res., vol. $2, \mathrm{n}^{\circ} 4$ (1966), 709-715.

Watsox (K. K.). - Experimental and numerical study of column drainatge. Proce. ASCE, $J$. of the Hydraulies Division, rol. 93, HY-2, (1967), I-15.

DE WIEST (R.J.M.), -- Geohydrology. J. Wiley and Sons, Ed. $(1965)$.

Youxcs (E.G.). - The drainage of liquids from porous materia1s. J. of Geophlys. Res., vol. 65, (1960), $4025-4030$.

Youxes (E.G.) et Smres (D. E.). - The pumping of water from wells in unconfined aquifers. A note on the applicability of Theis' formula. I. of Geophlys. Res., vol. 68, $(1963)$, 5 905-5 907 . 
Abstract

A study of transient flow conditions during the drainage of free-surface aquifers

by G. Vachaud *

The objective of the study was to examine the validity of some of the current assumptions and models used to describe the drainage of unconfined aquifers.

A $\gamma$-ray attenuation technique was used to follow rapid water content changes in the soil during drainage.

The first aim of drainage experiments was to evaluate the effect of time, depth of the water table and drawndown on the value of the storage coefficient. This coefficient has often been assumed to be constant and equal to the specific yield when considering problems of free-surface flow in ground water hydrodynamics.

Taking into account the flow of water in the unsaturated zone, it could be easily shown that the specific yield $\varepsilon$ has to be considered only as an asymptotic limit of the value of the storage coefficient $S$.

During the drainage of a water table aquifer initially at a certain depth $\mathrm{H}$, it is important to note that the maximum drainable volume of water $V_{\infty}$ will be given, per unit surface of soil, by the area limited by the drainage branches of the capillary pressures curves corresponding to the initial and final positions of the water table.

From the analysis of how the drained volume of water varies for different drawdown values and a water table initially at a soil surface, it is possible to make a comparative study of the drainage duration. Considering as a criterion that the experiments ended for the time $t_{99}$, for which $99 \%$ of the total drainable volume of water had flowed away, we obtained an empirical relation between $t_{99}$, drawdown $Z$ and saturated permeability $\mathrm{K}_{0}$.

An attempt was then made to adapt capillary models to drainage problems. We considered three series of models: - the gravity model, neglecting the unsaturated zone;

- the "single capillary" model, already developed by E. G. Youngs and considering the analogy between a unique family of capillary tubes whose diameter is defined by the capillary fringe of the soil, and the soil;

- the "multi-capillary" model, considering the analogy between a series of different families of capillary tubes and

the soil, the capillary rise in each family being equal to the capillary rise for the corresponding values of pore range.

Comparing experimental values of the drained volume of water with theoretical ones, obtained by analysis of capillary models, it is shown that the single capillary model is very satisfactory at the beginning of a drainage experiment. However, when the position of the maximum water content $\theta_{0}$ tends towards the capillary fringe corresponding to the equilibrium position of the drained water table, the multi-capillary model gives a good approximation of values of the drained volume of water.

We finally describe a method for determining the hydraulic conductivity function $k(\theta)$ from a transient flow analysis of the water content profiles. The generalized Darcy's law used to describe the flow of water in unsaturated soils is found to be valid. For different values of the water content $\theta, k$ is given by the ratio between unit discharge $q$ and capillary potential gradient.

It is necessary to emphasize the importance of the unsaturated zone between the water table and the soil surface in unconfined aquifer problems.

\footnotetext{
* Attaché de Recherches au C.N.R.S., Laboratoire de Mécanique des Fluides, Grenoble.
} 